\title{
NONCHARACTERISTIC EMBEDDINGS OF THE $n$-DIMENSIONAL TORUS IN THE $(n+2)$-DIMENSIONAL TORUS
}

\author{
DAVID MILLER
}

\begin{abstract}
We construct certain exotic embeddings of the $n$-torus $T^{n}$ in $T^{n+2}$ in the standard homotopy class. We turn an embedding $f: T^{n} \rightarrow T^{n+2}$ characteristic if there exists some map $\alpha: T^{n+2} \rightarrow T^{n+2}$ in the standard homotopy class with the property that $\alpha \circ f: T^{n} \rightarrow T^{n+2}$ is the standard coordinate inclusion and $\alpha\left(T^{n+2}-f\left(T^{n}\right)\right) \subset T^{n+2}-T^{n}$. We find examples of noncharacteristic embeddings, $f$, in dimensions $n=4 k+1, n \geq 5$, and show that these examples are not even cobordant to characteristic embeddings. We let $G$ denote the fundamental group of the complement of the standard coordinate inclusion, $T^{n+2}-T^{n}$. Then we can associate to $f$ a real-valued signature function on the set of $j$-dimensional unitary representations of $\bar{G}$, where $\bar{G}$ denotes the fundamental group of the localization of $T^{n+2}-T^{n}$ with respect to homology with local coefficients in $\mathbb{Z}\left[\mathbb{Z}^{n+2}\right]$. This function is a cobordism invariant which has certain periodicity properties for characteristic embeddings. We verify that this periodicity does not hold for our examples, $f$, implying that they are not characteristic. Additional results include a proof that the examples, $f$, become cobordant to characteristic embeddings upon taking the cartesian product with the identity map on a circle.
\end{abstract}

\section{INTRODUCTION}

In this paper we study embeddings of the $n$-torus $T^{n}$ in $T^{n+2}$ in the standard homotopy class. We develop cobordism invariants that detect whether these embeddings are characteristic in the sense that there exists a map $\alpha: T^{n+2} \rightarrow$ $T^{n+2}$, in the standard homotopy class, such that $\alpha \circ f: T^{n} \rightarrow T^{n+2}$ is the standard inclusion on coordinates and $\alpha\left(T^{n+2}-f\left(T^{n}\right)\right) \subset T^{n+2}-T^{n}$. We then construct noncharacteristic embeddings that are not even cobordant to characteristic embeddings.

Interest in the study of characteristic embeddings dates to the work of Browder and S. López de Medrano on embeddings in codimension $>2$, and of Cappell and Shaneson [CS1] in codimension 2. The study of characteristic embeddings arises naturally in codimension 2 in link theory [CS2]. An embedding $S^{n} \amalg \cdots \amalg S^{n} \rightarrow S^{n+2}$ is characteristic iff it is a boundary link in the sense that the embedded spheres bound disjoint hypersurfaces in $S^{n+2}$. It is a recent result of Cochran and Orr [CO1, $\mathrm{CO} 2]$ that there exist nonboundary links that are not cobordant to boundary links.

The present investigation of noncharacteristic embeddings of tori requires the solution of new algebraic and geometrical problems that do not arise in the

Received by the editors January 15, 1992.

1991 Mathematics Subject Classification. Primary 57Q60; Secondary 57Q45. 
case of links. These are related to the nontriviality of $\pi_{1}\left(T^{n}\right)$ and the fact that, unlike the situation in link theory, here $H_{1}\left(T^{n+2}-T^{n}\right) \rightarrow H_{1}\left(T^{n+2}\right)$ is an isomorphism. The general philosophy of studying characteristic embeddings is to compare them via homology surgery to the standard embeddings. For noncharacteristic embeddings the standard complements must be replaced by suitable localizations [CS2, LD, CO1, L3].

Sections 1-3 consist of definitions of the objects involved in the problem, and the construction of localization functors and algebraic closures. We construct a Vogel localization functor for the category of spaces equipped with maps to the $(n+2)$-dimensional torus $T^{n+2}$. This functor is universal with respect to maps inducing isomorphisms on homology with local coefficients in $\mathbb{Z}\left[\mathbb{Z}^{n+2}\right]$ and normal surjections on a subgroup of fundamental group. We then define and prove the existence of an algebraic closure for the category of groups equipped with surjections onto $\mathbb{Z}^{n+2}$. We show that for a $K(\pi, 1)$-space $X$, the fundamental group of the localization of the space is the algebraic closure of the group $\pi$. Finally, we associate to embeddings $f: T^{n} \rightarrow T^{n+2}$ a homomorphism from the fundamental group of the complement to a particular algebraic closure.

In $\S 4$, we construct certain quotient groups of the fundamental group of our target space for surgery: the localization of the complement of the standard coordinate inclusion, $T^{n+2}-T^{n}$. These quotient groups are still in the category of groups equipped with surjections onto $\mathbb{Z}^{n+2}$. We will pass to these quotients in order to perform computations.

In $\S 5$, we define signature functions on the set of $k$-dimensional unitary representations of a group $G$ associated to manifolds $M$ equipped with homomorphisms $\pi_{1}(M) \rightarrow G$. We discuss the cobordism invariance of these signature functions. We also associate signature functions on the set of $k$-dimensional unitary representations of $G$ to elements of the Cappell-Shaneson homology surgery obstruction groups associated to the group ring of $G$. Finally, we describe certain lines in the set of unitary representations of the quotient groups constructed in $\S 4$ and in the set of unitary representations of the algebraic closures of these groups and compare periodicities of signature functions restricted to these lines.

In $\S 6$, we construct examples of noncharacteristic embeddings in dimensions $n=4 k+1, n \geq 5$, by realizing certain elements of the homology surgery obstruction $\Gamma$-groups of Cappell and Shaneson [CS1]. We compute signatures for these embeddings at different points along a line of representations discussed in $\S 5$. We find that a certain periodicity necessary for an embedding to be characteristic fails to occur. Thus, these embeddings are examples of noncharacteristic embeddings. Since the signature is a cobordism invariant, these embeddings are not even cobordant to characteristic embeddings. In addition, we find that upon taking the cartesian product with the identity map on a circle, these examples become cobordant to characteristic embeddings.

The results presented here constitute my Ph.D. thesis written at the Courant institute of Mathematical Sciences of New York University under the supervision of Professor Sylvain Cappell. I would like to thank him for his help and his inspiring suggestions and explanations. I would also like to thank Professor Kent Orr of Indiana University for assisting me with the literature and ideas that compose the background of this work. 


\section{DEFINITIONS AND PRELIMINARIES}

We study piecewise linear locally flat embeddings $f: T^{n} \rightarrow T^{n+2}$ such that $f_{*}: \mathbb{Z}^{n} \rightarrow \mathbb{Z}^{n+2}$, where $f_{*}$ is the induced homomorphism on fundamental group, is the standard inclusion on the first $n$ coordinates. Two embeddings $f_{0}, f_{1}$ : $T^{n} \rightarrow T^{n+2}$ are said to be cobordant if there is a submanifold $V$ of $T^{n+2} \times I$, piecewise-linear homeomorphic to $T^{n} \times I$, meeting the boundary transversly, such that for $i=0,1$,

$$
V \cap\left(T^{n+2} \times i\right)=\operatorname{im}\left(f_{i}\right) .
$$

Although our discussion takes place in the PL category, the results we obtain also hold for smooth or topological locally flat embeddings.

Definition 1.1. An embedding $f$ of $T^{n}$ in $T^{n+2}$ is said to be characteristic if there is a map $\alpha: T^{n+2} \rightarrow T^{n+2}$ homotopic to the identity, such that $\alpha \circ f$ : $T^{n} \rightarrow T^{n+2}$ is the standard inclusion of tori and $\alpha\left(T^{n+2}-f\left(T^{n}\right)\right) \subset T^{n+2}-T^{n}$.

Definition 1.2. Two manifolds $M_{1}, M_{2}$ equipped with $\mathbb{Z}\left[\mathbb{Z}^{n+2}\right]$-coefficients are called $\mathbb{Z}\left[\mathbb{Z}^{n+2}\right]$-homology bordant if there is a manifold $W$, equipped with $\mathbb{Z}\left[\mathbb{Z}^{n+2}\right]$ coefficients, such that $\partial W=M_{1}-M_{2}$, and

$$
H_{*}\left(W, M_{1} ; \mathbb{Z}\left[\mathbb{Z}^{n+2}\right]\right)=H_{*}\left(W, M_{2} ; \mathbb{Z}\left[\mathbb{Z}^{n+2}\right]\right)=0
$$

in local coefficients.

Let $D$ be a group equipped with a surjection $\phi: D \rightarrow \mathbb{Z}^{n+2}$. We term a manifold $M$ a $D$-manifold if it is equipped with a homomorphism $\alpha: \pi_{1}(M) \rightarrow D$ such that $\phi \circ \alpha$ is a surjection. We denote it $(M, \alpha)$.

Definition 1.3. We call two $D$-manifolds $\left(M_{1}, \alpha_{1}\right)$ and $\left(M_{2}, \alpha_{2}\right)$ equipped with $\mathbb{Z}\left[\mathbb{Z}^{n+2}\right]$ coefficients $\mathbb{Z}\left[\mathbb{Z}^{n+2}\right]$-homology D-bordant if they are $\mathbb{Z}\left[\mathbb{Z}^{n+2}\right]$ homology bordant via a $D$-manifold $(W, \beta)$, equipped with $\mathbb{Z}\left[\mathbb{Z}^{n+2}\right]$ coefficients, such that $\alpha_{1}=\beta \circ\left(i_{1}\right)_{*}$, and $\alpha_{2}=\beta \circ\left(i_{2}\right)_{*}$, where $i_{1}, i_{2}$ are inclusions.

Let $M(f)$ denote the closure of the complement of a tubular neighborhood of the embedding $f$.

Proposition 1.1. If $f_{1}, f_{2}$ are cobordant embeddings of $T^{n}$ in $T^{n+2}$, then $M\left(f_{1}\right)$ and $M\left(f_{2}\right)$ are $\mathbb{Z}\left[\mathbb{Z}^{n+2}\right]$-homology bordant in the local coefficient system inherited from $T^{n+2}$.

Proof. In $T^{n+2}$, a path $\alpha_{x y}$ from $x$ to $y$ gives an isomorphism of $\pi_{1}\left(T^{n+2}, x\right)$ and $\pi_{1}\left(T^{n+2}, y\right)$ by $\left(\alpha_{x y}\right)_{*}\left(\beta_{x}\right)=\alpha_{x y}^{-1} \beta_{x} \alpha_{x y}$. This induces a system of local coefficients over $\mathbb{Z}\left[\mathbb{Z}^{n+2}\right]$ that is simple in the sense that if $\alpha_{x x}$ is any closed path then it induces the identity isomorphism of $\mathbb{Z}\left[\mathbb{Z}^{n+2}\right]$. This is because $\mathbb{Z}^{n+2}$ is abelian, and

$$
\left(\alpha_{x x}\right)_{*}\left(\beta_{x}\right) \stackrel{\text { def }}{=}\left(\alpha_{x x}\right)^{-1} \beta_{x} \alpha_{x x}=\beta_{x}, \quad \beta_{x} \in \pi_{1}\left(T^{n+2}, x\right)=\mathbb{Z}^{n+2} .
$$

As a result, the isomorphism $\left(\alpha_{x y}\right)_{*}$ is independent of path. So we can think of our system as coefficients in a fixed ring $\mathbb{Z}\left[\mathbb{Z}^{n+2}\right]$ and apply excision. Let

$$
A_{i} \stackrel{\text { def }}{=} N(V) \cup T^{n+2} \times i, \quad i=0,1,
$$


where $N(V)$ is a closed tubular neighberhood of $V$. Let $U=\stackrel{\circ}{N}(V)$ be the interior of $N(V)$. Then $M\left(f_{i}\right)$ is a deformation retract of $A_{i}-U$ and we find that

$$
\begin{aligned}
H_{*}\left(T^{n+2} \times I-U, M\left(f_{i}\right) ; \mathbb{Z}\left[\mathbb{Z}^{n+2}\right]\right) & =H_{*}\left(T^{n+2} \times I-U, A_{i}-U ; \mathbb{Z}\left[\mathbb{Z}^{n+2}\right]\right) \\
& =H_{*}\left(T^{n+2} \times I, A_{i} ; \mathbb{Z}\left[\mathbb{Z}^{n+2}\right]\right) \\
& =H_{*}\left(T^{n+2} \times I, T^{n+2} \times i ; \mathbb{Z}\left[\mathbb{Z}^{n+2}\right]\right)=0
\end{aligned}
$$

for $i=0,1$, where the second isomorphism is excision and the other two are by virtue of deformation retracts. This result says that $\left(T^{n+2} \times I-U, M\left(f_{1}\right), M\left(f_{2}\right)\right)$ is a $\mathbb{Z}\left[\mathbb{Z}^{n+2}\right]$-homology bordism.

In the following let $X=T^{n+2}-T^{n}$ be the complement of the standard embedding. Let $G=\pi_{1}(X)=\mathbb{Z}^{n} \times F_{2}$. Let the generators of $G$ be $t_{1}, \ldots, t_{n}$ for the free abelian factor, and $a, b$ for the free factor.

\section{LOCALIZATION OF SPACES AND GROUPS}

Adapting the theory and notation of Vogel [V], let $\mathscr{C}$ be the category of pointed CW complexes over $T^{n+2}$. An object of $\mathscr{C}$ is a map $\varphi: Y \rightarrow T^{n+2}$ where $Y$ has the homotopy type of a pointed $\mathrm{CW}$ complex, and $\varphi$ induces a surjection on fundamental group. A morphism of $\mathscr{C}$ from $\varphi: Y \rightarrow T^{n+2}$ to $\psi: Z \rightarrow T^{n+2}$ is a map $h: Y \rightarrow Z$ such that $\varphi=\psi \circ h$, and will be called a $T^{n+2}$-map. Let $\mathscr{W}$ be the class of $T^{n+2}$-maps of finite $\mathrm{CW}$ complexes inducing isomorphisms of homology over the obvious system of local coefficients in $\mathbb{Z}\left[\mathbb{Z}^{n+2}\right]$ and normal surjections on the subgroups of fundamental group associated to the $T^{n+2}$-structure. The latter condition would mean, for example, that if the above map $h$ were in $\mathscr{W}$, then $\operatorname{ker}\left(\psi_{*}\right)$ would be generated by $h_{*}\left(\operatorname{ker}\left(\phi_{*}\right)\right)$ and products of conjugates thereof.

Definition 2.1. Let $\overline{\mathscr{W}}$ be the class of $T^{n+2}$ maps $X \rightarrow Y$ such that for any commutative diagram

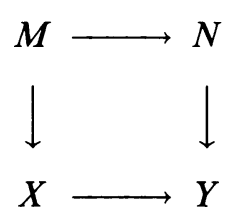

where $M \rightarrow N$ is a map between finite $\mathrm{CW}$ complexes, there is a factorization:

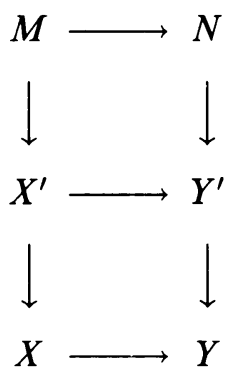

where $X^{\prime}$ and $Y^{\prime}$ are finite $\mathrm{CW}$ complexes and $X^{\prime} \rightarrow Y^{\prime}$ is in $\mathscr{W}$.

Thus the class of maps $\overline{\mathscr{W}}$ includes maps between $\mathrm{CW}$ complexes that are not necessarily finite. 
Definition 2.2. A localization functor for the class $\overline{\mathscr{W}}$ is a functor $E: \mathscr{C} \rightarrow$ $\mathscr{C}$, endowed with a natural transformation $\eta: 1 \rightarrow E$ satisfying the following conditions:

(1) For any $Y \in \mathscr{C}, \eta_{Y}: Y \rightarrow E(Y)$ is in the class $\overline{\mathscr{W}}$.

(2) For any $Y \in \mathscr{C}$, and any map $h: Y \rightarrow Z$ in $\overline{\mathscr{W}}$, there exists a map $\omega: Z \rightarrow E(Y)$ such that $\eta_{Y}=\omega \circ h$.

Often we will use the notation $E_{Y}$ for $\eta_{Y}$.

Proposition 2.1 [V]. There exists a localization functor for the class $\overline{\mathscr{W}}$. Thus, if $Y$ is a space in $\mathscr{C}$, it has a localization, $E(Y)$, that is unique up to natural equivalence in $\mathscr{C}$. Occasionally, we will use the notation $\bar{Y}$ for the $\overline{\mathscr{W}}$-localization of $Y$.

Similarly, we define localization of groups. Let $\mathscr{G}$ be the category of groups over $\mathbb{Z}^{n+2}$. An object of $\mathscr{G}$ is a map $\varphi: D \rightarrow \mathbb{Z}^{n+2}$, where $D$ is a group and $\varphi$ is a surjective homomorphism. A morphism of $\mathscr{G}$ from $\varphi: D \rightarrow \mathbb{Z}^{n+2}$ to $\psi: F \rightarrow \mathbb{Z}^{n+2}$ is a homomorphism $h: D \rightarrow F$ such that $\varphi=\psi \circ h$. Let $\mathscr{H}$ be the class of homomorphisms between finitely presented groups in the category $\mathscr{G}$ that are normal surjections on the subgroups associated to the $\mathbb{Z}^{n+2}$-structures and induce isomorphisms on $H_{1}$ and surjections on $H_{2}$ where this denotes homology over the obvious system of local coefficients in $\mathbb{Z}\left[\mathbb{Z}^{n+2}\right]$.

Definition 2.3. Let $\overline{\mathscr{H}}$ be the class of homomorphisms $D \rightarrow F$ in $\mathscr{G}$ such that for any commutative diagram of groups and homomorphisms

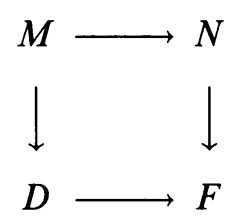

where $M$ and $N$ are finitely presented groups, there is a factorization

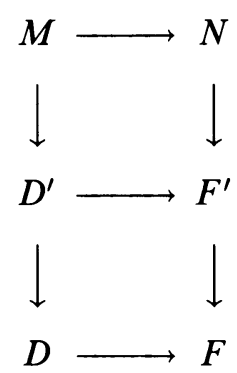

where $D^{\prime}$ and $F^{\prime}$ are finitely presented groups and $D^{\prime} \rightarrow F^{\prime}$ is in $\mathscr{H}$.

Definition 2.4. A localization functor for the class $\overline{\mathscr{H}}$ is a functor $E: \mathscr{G} \rightarrow \mathscr{G}$ endowed with a natural transformation $\eta: 1 \rightarrow E$ satisfying (1) and (2) of Definition 2.2 for $\mathscr{G}$ and $\overline{\mathscr{H}}$. We will often use $E_{D}$ for $\eta_{D}$.

Proposition 2.2. There exists a localization functor $E$ for the class $\overline{\mathscr{H}}$, unique up to natural equivalence. In fact, if $D$ is a group, then its localization is determined up to natural isomorphism.

Proof. In [LD], a Vogel localization functor for CW complexes with respect to maps inducing isomorphisms on homology with integral coefficients and normal 
surjections on fundamental groups is developed. We denote this by $E^{\mathbb{Z}}$. Its construction is analogous to the above construction of the localization functor for the class of maps $\overline{\mathscr{W}}$. If $X$ is a space, $E^{\mathbb{Z}} X$ is well defined up to homotopy type. If $G$ is a group, we define a localization functor $E_{*}^{\mathbb{Z}}$ for groups with respect to homology with integral coefficients by

$$
E_{*}^{\mathbb{Z}}(G) \stackrel{\text { def }}{=} \pi_{1} E^{\mathbb{Z}} K(G, 1), \quad\left(E_{*}^{\mathbb{Z}}\right)_{G} \stackrel{\text { def }}{=}\left(\left(E^{\mathbb{Z}}\right)_{K(G, 1)}\right)_{*}: G \rightarrow E_{*}^{\mathbb{Z}}(G) .
$$

We verify that this is a localization functor for groups.

(i) Since $E_{K(G, 1)}^{\mathbb{Z}}: K(G, 1) \rightarrow E^{\mathbb{Z}} K(G, 1)$ is a normal surjection and induces isomorphisms on $H_{1},\left(E_{*}^{\mathbb{Z}}\right)_{G}: G \rightarrow E_{*}^{\mathbb{Z}}(G)$ is a normal surjection on fundamental group and induces isomorphisms on $H_{1}$ of groups, because in both cases $H_{1}$ is $\pi_{1}$ abelianized. Now,

$$
\left(E_{K(G, 1)}^{\mathbb{Z}}\right)_{*}: H_{2}(K(G, 1)) \rightarrow H_{2}\left(E^{\mathbb{Z}} K(G, 1)\right)
$$

is an isomorphism. Hopf's theorem tells us there is a natural surjection

$$
q: H_{2}\left(E^{\mathbb{Z}} K(G, 1)\right) \rightarrow H_{2}\left(\pi_{1}\left(E^{\mathbb{Z}} K(G, 1)\right)\right) \stackrel{\text { def }}{=} H_{2}\left(E_{*}^{\mathbb{Z}}(G)\right) .
$$

Thus,

$$
\left(\left(E_{*}^{\mathbb{Z}}\right)_{G}\right)_{*}: H_{2}(G) \rightarrow H_{2}\left(E_{*}^{\mathbb{Z}}(G)\right)
$$

is a surjection.

(ii) Universality. If $h: \pi \rightarrow \pi^{\prime}$ is a normal surjection between finitely presented groups and induces an isomorphism on $H_{1}$ and a surjection on $H_{2}$ with integral coefficients, we will demonstrate that there is a homomorphism $g: \pi^{\prime} \rightarrow E_{*}^{\mathbb{Z}}(\pi)$ with the same properties such that $\left(E_{*}^{\mathbb{Z}}\right)_{\pi}=g \circ h$. We accomplish this by constructing a space $V$ with fundamental group $\pi^{\prime}$ and a map $F: K(\pi, 1) \rightarrow V$ inducing integral homology isomorphisms. Then universality of $E^{\mathbb{Z}}$ will give universality of $E_{*}^{\mathbb{Z}}$.

Let $\overline{V^{(2)}}$ be the 2-skeleton of $K\left(\pi^{\prime}, 1\right)$. Let $F^{(1)}$ be the restriction of $\mathbf{h}$ : $K(\pi, 1) \rightarrow K\left(\pi^{\prime}, 1\right)$ to 1 -skeleta. Let $\left\{\varphi_{\alpha}\right\}$ be attaching maps for the 2-cycles generating $H_{2}(\pi)$. For each generator $\gamma$ of $H_{2}\left(\overline{V^{(2)}}\right)$ choose an element $\beta$ of $H_{2}(K(\pi, 1))$ such that $\left(F^{(1)}\right)_{*}(\beta)=\gamma$. Let $\left\{\varphi_{\beta}\right\}$ be a set of attaching maps for representatives of these classes. Let $\left\{\mathbf{h} \circ \varphi \mid \varphi \in\left\{\varphi_{\alpha}\right\}, \varphi \notin\left\{\varphi_{\beta}\right\}\right\}$ be attaching maps for additional 2-cells to $\overline{V^{(2)}}$. Call the resulting space $V^{(2)}$ and the resulting map $F^{(2)}: K(\pi, 1)^{(2)} \rightarrow V^{(2)}$. Proceed inductively to obtain an $H_{*}$ isomorphism: $F: K(\pi, 1) \rightarrow V$. Then by the universality of $E^{\mathbb{Z}}$ there is a map $G: V \rightarrow E^{\mathbb{Z}}(K(\pi, 1))$. Let

$$
g \stackrel{\text { def }}{=} G_{*}: \pi_{1} V \rightarrow \pi_{1} E^{\mathbb{Z}}(K(\pi, 1)) .
$$

In fact, $g: \pi^{\prime} \rightarrow E_{*}^{\mathbb{Z}}(\pi) . \quad g$ is a normal surjection by construction of the functor $E^{\mathbb{Z}}$. Moreover, $g$ is an isomorphism on $H_{1}$, since $H_{1}$ is the same for groups as for spaces. This means that $g$ produces a surjection onto the commutator relations of $E_{*}^{\mathbb{Z}}(\pi)$ and hence a surjection onto $H_{2}\left(E_{*}^{\mathbb{Z}}(\pi)\right)$. This verifies the universality of $E_{*}^{\mathbb{Z}}$.

We can use $E_{*}^{\mathbb{Z}}$ to define a localization functor for the class $\overline{\mathscr{H}}$. If $G \stackrel{\varphi}{\rightarrow} \mathbb{Z}^{n+2}$ is an object of $\mathscr{G}$, let $K=\operatorname{ker}(\varphi)$. Let $l: K \rightarrow E_{*}^{\mathbb{Z}}(K)$. Then $E_{*}^{\mathbb{Z}}(K)$ is the normal closure of the image of $l$, i.e., all elements of $E_{*}^{\mathbb{Z}}(K)$ are products of conjugates of elements in $\operatorname{im}(l)$. Thus, the extension $K \mapsto G \rightarrow \mathbb{Z}^{n+2}$ allows 
us to define a localization functor $E$ for groups via the extension $E_{*}^{\mathbb{Z}}(K) \longmapsto$ $E(G) \rightarrow \mathbb{Z}^{n+2}$ where multiplication of elements in $E(G)$ is induced by multiplication in $G$. Since $E_{*}^{\mathbb{Z}}(K)$ is unique up to natural isomorphism, so is $E(G)$ constructed in this way.

\section{Algebraic Closures AND STRUCTURES ON EMBEDDINGS}

We adapt the methods of Levine [L2]. Let $D$ be a group in $\mathscr{G}$, i.e., there is a surjective homomorphism $\varphi: D \rightarrow \mathbb{Z}^{n+2}$. Let $K=\operatorname{ker}(\varphi)$. An element $w=w\left(x_{1}, x_{2}, \ldots, x_{n}\right) \in K * F_{m}$ where $F_{m}=F\left(x_{1}, \ldots, x_{m}\right)$ is the free group on $m$ letters, will be called a $\mathbb{Z}^{n+2}$-monomial over $D$. A $\mathbb{Z}^{n+2}$-monomial over $D$ is termed $\mathbb{Z}^{n+2}$-trivial if $w$ lies in the kernel of the projection $K * F_{m} \rightarrow F_{m}$. Clearly a monomial is $\mathbb{Z}^{n+2}$-trivial when it is a product of conjugates of elements of $K$. A system of equations over $D$ :

$$
x_{i}=w_{i}\left(x_{1}, x_{2}, \ldots, x_{m}\right), \quad i=1, \ldots, m,
$$

is termed $\mathbb{Z}^{n+2}$-trivial if each $w_{i}$ is a $\mathbb{Z}^{n+2}$-trivial monomial over $D$.

Definition 3.1. A group $C$ in $\mathscr{G}$ is termed $\mathbb{Z}^{n+2}$-algebraically closed if every $\mathbb{Z}^{n+2}$-trivial system of equations over $C$ has a unique solution in $C$.

Definition 3.2. A $\mathscr{G}$-homomorphism $h: D \rightarrow C$ where $C$ is $\mathbb{Z}^{n+2}$ algebraically closed will be termed a $\mathbb{Z}^{n+2}$-algebraic closing of $D$.

Definition 3.3. If $D$ is a group in $\mathscr{G}$, a $\mathbb{Z}^{n+2}$-algebraic closure of $D$ is a $\mathbb{Z}^{n+2}$ algebraic closing $h: D \rightarrow C$ which satisfies the following universality property: for any $\mathbb{Z}^{n+2}$-algebraic closing $g: D \rightarrow B$ there exists a unique homomorphism $\rho: C \rightarrow B$ such that $\rho \circ h=g$.

Proposition 3.1. For every group $D$ in $\mathscr{G}$ there exists a $\mathbb{Z}^{n+2}$-algebraic closure of $D$ which is unique up to isomorphism. (Two algebraic closings $\phi: D \rightarrow F$ and $\psi: D \rightarrow F^{\prime}$ of $D$ are said to be isomorphic if there is a group isomorphism $\gamma: F \rightarrow F^{\prime}$ such that $\psi=\gamma \circ \phi$.) We will denote it by $\bar{D}$.

Proof. It is a result of Levine [L2] that every group $K$ has an algebraic closure $\hat{K}$ in the sense that every system of equations $x_{i}=w_{i}\left(x_{1}, \ldots, x_{m}\right)$, $i=1, \ldots, m$, where $w_{i} \in K * F_{m}=K * F\left(x_{1}, \ldots, x_{m}\right)$ and $w_{i}$ is in the kernel of the obvious projection $K * F_{m} \rightarrow F_{m}$, has a unique solution in $\hat{K}$. Such monomials are termed contractible. Clearly, a monomial is contractible when it is a product of conjugates of elements of $K$. It is also shown in [L2] that $\hat{K}$ has the universal property of Definition 3.3 with respect to algebraic closings and that the inclusion $K \rightarrow \hat{K}$ is a normal surjection. Thus the extension $K \mapsto D \rightarrow \mathbb{Z}^{n+2}$ allows us to define $\bar{D}$ via the extension $\hat{K} \mapsto \bar{D} \rightarrow \mathbb{Z}^{n+2}$. Since $\hat{K}$ is the normal closure of $K \triangleleft \hat{K}$, the group structure on $\bar{D}$ is induced by the group structure on $D$ in the obvious manner. It is clear that $\hat{K}$ is unique up to natural isomorphism. Thus, $\bar{D}$ defined in this manner is also.

Proposition 3.2. If $\eta_{D}: D \rightarrow E(D)$ is $\overline{\mathscr{H}}$ localization of the group $D$, then $E(D)$ is the $\mathbb{Z}^{n+2}$-algebraic closure of $D$.

Proof. Let $\varphi: D \rightarrow \mathbb{Z}^{n+2}$ and let $F \stackrel{\text { def }}{=} \operatorname{ker}(\varphi)$. It suffices to show that the $E^{\mathbb{Z}}$ localization of a group yields the ordinary algebraic closure of the group. Recall 
that if $F$ is a group,

$$
E_{*}^{\mathbb{Z}}(F) \stackrel{\text { def }}{=} \pi_{1} E^{\mathbb{Z}}(K(F, 1))
$$

Denote $K(F, 1)$ by $Y$.

(i) In constructing the $E^{\mathbb{Z}}$ localization of the space $Y$, we attach pairs of finite CW-complexes $(K, L)$, where $K / L$ is contractible, to $Y$ :

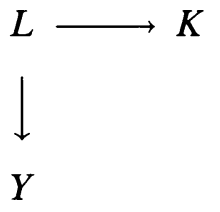

Now $\pi_{1}\left(Y \cup_{L} K\right)$ is the normal closure of the image of $\pi_{1} Y$ under inclusion. In fact, $\pi_{1}\left(Y \cup_{L} K\right)$ is generated by $i_{*}\left(\pi_{1} Y\right)$ and a finite number of additional elements $\left\{x_{i}\right\}$. Thus, each such attachment corresponds to adjoining to $\pi_{1} Y$ the solutions to a system of monomial equations $x_{i}=w_{i}\left(x_{1}, \ldots, x_{m}\right), i=$ $1, \ldots, m$, where $w_{i} \in \pi_{1} Y * F_{m}$ is in the kernel of the projection $\pi_{1} Y * F_{m} \rightarrow$ $F_{m}$.

(ii) Consider any system of equations $x_{i}=w_{i}\left(x_{1}, \ldots, x_{m}\right), i=1, \ldots, m$, where $w_{i} \in \pi_{1} Y * F_{m}$ is in the kernel of the projection $\pi_{1} Y * F_{m} \rightarrow F_{m}$. Let $G_{S}$ be the group obtained from $\pi_{1} Y$ by attaching generators $x_{1}, \ldots, x_{m}$ and relations $x_{i}=w_{i}\left(x_{1}, \ldots, x_{m}\right), i=1, \ldots, m$. Let

$$
Y_{S}=Y \cup_{x_{0}}\left(e_{1}^{1} \cup \cdots \cup e_{1}^{m}\right) \cup_{\varphi_{\alpha}}\left(e_{2}^{1} \cup \cdots \cup e_{2}^{m}\right)
$$

by adjoining 1-cells and 2-cells such that $G_{S}=\pi_{1}\left(Y_{S}\right)$. Let

$$
K=\left(\bigcup e_{1}^{i}\right) \cup_{\varphi_{\alpha} \mid}\left(\bigcup e_{2}^{i}\right), \quad L=\bigcup\left(\partial e_{2}^{i}\right)-\bigcup\left(e_{1}^{i}\right)
$$

Then $K / L$ is a 1-point wedge of discs, and we have the diagram

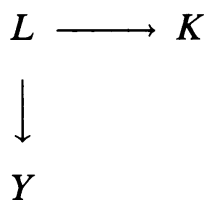

We see that $Y_{S}=Y \cup_{L} K$ and that $(K, L)$ is attached during $E^{\mathbb{Z}}$ localization of $Y$. Thus the solutions to any contractible system over $\pi_{1} Y$ are adjoined by $E^{\mathbb{Z}}$ localization.

Together, (i) and (ii) imply that

$$
\widehat{\pi_{1} Y}=\pi_{1}\left(E_{*}^{\mathbb{Z}}(Y)\right)
$$

or, simply put, that $\hat{F}=E_{*}^{\mathbb{Z}}(F)$. Then, by definition $\bar{D}=E(D)$.

Henceforth, we will use $\bar{D}$ interchangeably to denote the $\mathbb{Z}^{n+2}$-algebraic closure and the $\overline{\mathscr{H}}$-localization of the group $D$.

If $f: T^{n} \rightarrow T^{n+2}$ is an embedding in the standard homotopy class, and $G=\mathbb{Z}^{n} \times F_{2}$ as above, we investigate ways of making $M(f)$ a $\bar{G}$-manifold. Let $x_{1}, \ldots, x_{n}, x_{n+1}$ be generators of $\pi_{1}(\partial M(f)) \cong \mathbb{Z}^{n+1}$. Let $i: \partial(M(f)) \hookrightarrow$ $M(f)$ be the inclusion of the boundary. Let

$$
m_{i}=i_{*}\left(x_{i}\right) \in \pi_{1}(M(f)), \quad i=1, \ldots, n .
$$


Let $m_{n+1}, m_{n+2} \in \pi_{1}(M(f))$ be any choice of elements such that if

$$
h: \pi_{1}(M(f)) \rightarrow H_{1}(M(f)) \cong \mathbb{Z}^{n+2}
$$

is abelianization, then $h\left(m_{1}\right), \ldots, h\left(m_{n+1}\right), h\left(m_{n+2}\right) \in H_{1}(M(f))$ is a set of generators. . Let $\mu_{1}, \ldots, \mu_{n+2}$ be a set of loops representing $m_{1}, \ldots$, $m_{n+2}$ respectively. We interchangeably term $\left\{\mu_{i}\right\}$ and $\left\{m_{i}\right\}$ a choice of meridians for $f$.

Definition 3.4. We term a homomorphism $\alpha: \pi_{1}(M(f)) \rightarrow \bar{G}$ a $\bar{G}$-structure for $M(f)$ if $\alpha\left(m_{i}\right)$ is a conjugate of $t_{i}$ in $\bar{G}, i=1, \ldots, n, \alpha\left(m_{n+1}\right)$ is a conjugate of $a$, and $\alpha\left(m_{n+2}\right)$ is a conjugate of $b$ in $\bar{G}$. If $M(f)$ is equipped with such a structure, $f$ will be termed a $\bar{G}$-embedding.

Proposition 3.3. If $f: T^{n} \rightarrow T^{n+2}$ is an embedding in the standard homotopy class, $n>1$, then $M(f)$ has a $\bar{G}$-structure for any choice of meridians $\left\{m_{i}\right\}$. Two $\bar{G}$-structures $\alpha, \alpha^{\prime}$ for $\left\{m_{i}\right\}$ on $M(f)$ differ by an element of $\mathscr{A}(\bar{G})$, the group of automorphisms of $\bar{G}$ which conjugate each $t_{i}$ and $x_{i}$.

Proof. Define $\tau: G \rightarrow \pi_{1}(M(f))$ by stipulating that $\tau\left(t_{i}\right)=m_{i}, i=1, \ldots, n$, $\tau(a)=m_{n+1}$, and $\tau(b)=m_{n+2}$. This is a homomorphism as $\left.\tau\right|_{\mathbb{Z}^{n}}$ coincides with $\left.i_{*}\right|_{\mathbb{Z}^{n}}$, while

$$
\left[m_{i}, m_{n+1}\right]=\left[m_{i}, m_{n+2}\right]=e, \quad i=1, \ldots, n,
$$

because for $i=1, \ldots, n, \mu_{i} \vee_{x_{0}} \mu_{n+1}$ and $\mu_{i} \vee_{x_{0}} \mu_{n+2}$ can be filled in, in $T^{n+2}$, by 2 -discs which, by general position arguments, can be made to lie in $M(f)$ and to intersect $\partial M(f)$ only on $\mu_{i}$ and at points.

Recall from the proof of Proposition 1.1 that homology with local coefficients in $\mathbb{Z}\left[\mathbb{Z}^{n+2}\right]$ of $M(f)$ satisfies excision. It follows that

$$
\begin{aligned}
H_{1}\left(\pi_{1}(M(f)) ; \mathbb{Z}\left[\mathbb{Z}^{n+2}\right]\right) & \cong H_{1}\left(M(f) ; \mathbb{Z}\left[\mathbb{Z}^{n+2}\right]\right) \cong H_{1}\left(X ; \mathbb{Z}\left[\mathbb{Z}^{n+2}\right]\right) \\
& \cong H_{1}\left(\pi_{1} X ; \mathbb{Z}\left[\mathbb{Z}^{n+2}\right]\right)=\mathbb{Z}[\mathbb{Z}] \oplus \mathbb{Z}[\mathbb{Z}]
\end{aligned}
$$

while

$$
H_{2}\left(M(f) ; \mathbb{Z}\left[\mathbb{Z}^{n+2}\right]\right)=H_{2}\left(X ; \mathbb{Z}\left[\mathbb{Z}^{n+2}\right]\right)=0
$$

which, by Hopf's theorem, implies that

$$
H_{2}\left(\pi_{1}(M(f)) ; \mathbb{Z}\left[\mathbb{Z}^{n+2}\right]\right)=0 .
$$

Obviously, this means that $\tau_{*}: H_{2}\left(G ; \mathbb{Z}\left[\mathbb{Z}^{n+2}\right]\right) \rightarrow H_{2}\left(\pi_{1}(M(f)) ; \mathbb{Z}\left[\mathbb{Z}^{n+2}\right]\right)$ is a surjection. Now $m_{n+1}, m_{n+2}$ act on the generator $1 \oplus 1$ of

$$
H_{1}\left(\pi_{1}(M(f)) ; \mathbb{Z}\left[\mathbb{Z}^{n+2}\right]\right)
$$

as $\mathbb{Z} \times \mathbb{Z}$. Thus, $\tau_{*}: H_{1}\left(G ; \mathbb{Z}\left[\mathbb{Z}^{n+2}\right]\right) \rightarrow H_{1}\left(\pi_{1}(M(f)) ; \mathbb{Z}\left[\mathbb{Z}^{n+2}\right]\right)$ is an isomorphism.

As a result, the localization functor $\overline{\mathscr{H}}$ gives us a homomorphism $\alpha$ : $\pi_{1}(M(f)) \rightarrow \bar{G}$ such that $E_{G}=\alpha \circ \tau$. Clearly, $\alpha$ is a $\bar{G}$-structure for $f$.

Let $\alpha, \alpha^{\prime}$ be two such structures for $\left\{m_{i}\right\}$. Then they differ by an automorphism of $\bar{G}$ that conjugates $t_{1}, \ldots, t_{n}, a, b$.

Proposition 3.4. If two $\bar{G}$-embeddings, $f_{0}, f_{1}$ are cobordant with meridians $\left\{m_{i}^{0}\right\}$ and $\left\{m_{i}^{1}\right\}$ compatible under the inclusion in the bordism of the complements, then after a change of $\bar{G}$-structure, their complements are $\mathbb{Z}\left[\mathbb{Z}^{n+2}\right]$ homology $\bar{G}$-bordant. 
Proof. By Proposition 1.1, $M\left(f_{0}\right)$ and $M\left(f_{1}\right)$ are $\mathbb{Z}\left[\mathbb{Z}^{n+2}\right]$-homology bordant. This means that there is a triple $\left(W, M\left(f_{0}\right), M\left(f_{1}\right)\right)$ such that the inclusions $i_{0}$ and $i_{1}$ of $M\left(f_{0}\right)$ and $M\left(f_{1}\right)$ are isomorphisms on homology over the local coefficient systems. This means that by universality of the $\overline{\mathscr{H}}$ localization functor, there is a homomorphism $\beta: \pi_{1} W \rightarrow \bar{G}$ such that if $\alpha_{0}$ is the $\bar{G}$ structure for $M\left(f_{0}\right)$, then $\alpha_{0}=\beta \circ i_{0}$. The structure $\alpha_{0}$ presumes a choice of meridians $\left\{m_{i}^{0}\right\}$ for $f_{0}$. Since $i_{1}$ is an $H_{*}\left(; \mathbb{Z}\left[\mathbb{Z}^{n+2}\right]\right)$ isomorphism, we can choose $\left\{m_{i}^{1}\right\}$ such that $\left(i_{0}\right)_{*}\left(m_{i}^{0}\right)=\left(i_{1}\right)_{*}\left(m_{i}^{1}\right)$. Then we get a $\bar{G}$-structure $\alpha_{1}$ for $\left\{m_{i}^{1}\right\}$ on $M\left(f_{1}\right)$. After a change of $\bar{G}$ structure on $M\left(f_{1}\right)$ by an element of $\mathscr{A}(\bar{G})$, the triple $\left((W, \beta),\left(M\left(f_{0}\right), \alpha_{0}\right),\left(M\left(f_{1}\right), \alpha_{1}\right)\right)$ becomes a $\mathbb{Z}\left[\mathbb{Z}^{n+2}\right]$-homology $\bar{G}$-bordism.

\section{QUOTIENTS OF $G$ OVER ITS ABELIANIZATION}

To perform calculations we adapt the philosophy of Cochran and Orr [CO2] and construct explicit quotients of

$$
G \cong \mathbb{Z}^{n} \times F_{2}
$$

that are still objects in the category $\mathscr{G}$. We define an infinite group:

$$
Q \stackrel{\text { def }}{=}\left\langle x, y \mid[x, y]^{2}=e,[x,[x, y]]=[y,[x, y]]=e\right\rangle .
$$

Let $F_{2} \stackrel{\text { def }}{=}\langle a, b\rangle$ and define a homomorphism $\varphi: F_{2} \rightarrow Q$ by letting $\varphi(a)=x$ and $\varphi(b)=y$. Let $F \stackrel{\text { def }}{=} \operatorname{ker}(\varphi)$. So we consider the extension $F \hookrightarrow F_{2} \stackrel{\varphi}{\rightarrow} Q$. Let $F^{(1)} \stackrel{\text { def }}{=}[F, F]$. So, $F^{(1)} \triangleleft F \triangleleft F_{2}$, and $H_{1}(F)=F / F^{(1)}$. We now have the extension $H_{1}(F) \nrightarrow F_{2} / F^{(1)} \rightarrow Q$.

We describe $H_{1}(F)$ by studying the chain complex of the covering space, $K(F, 1)$, of the figure-eight, $K\left(F_{2}, 1\right)$, induced by $\varphi$ :

$$
C_{0}(K(F, 1))=\mathbb{Z}[Q], \quad C_{1}(K(F, 1))=\mathbb{Z}[Q] \oplus \mathbb{Z}[Q] .
$$

This is because $K\left(F_{2}, 1\right)$ has one 0 -cell and two 1-cells. The boundary map $\partial_{1}: C_{1} \rightarrow C_{0}$ is described by the 2 by 1 matrix

$$
\left(\begin{array}{l}
x-1 \\
y-1
\end{array}\right)
$$

So

$$
H_{1}(F) \cong \operatorname{ker}(\mathbb{Z}[Q] \oplus \mathbb{Z}[Q] \stackrel{(x-1, y-1)}{\longrightarrow} \mathbb{Z}[Q]) .
$$

We form a further quotient of $F_{2}$ by taking the quotient of $F_{2} / F^{(1)}$ by the kernel of the map

$$
\begin{aligned}
\left(q_{1}\right)_{*}: & \operatorname{ker}(\mathbb{Z}[Q] \oplus \mathbb{Z}[Q] \stackrel{(x-1, y-1)}{\longrightarrow} \mathbb{Z}[Q]) \\
& \rightarrow \operatorname{ker}(\mathbb{Z}[1, i, j, k] \oplus \mathbb{Z}[1, i, j, k] \stackrel{(i-1, j-1)}{\longrightarrow} \mathbb{Z}[1, i, j, k]) .
\end{aligned}
$$

We denote this extension as $H_{1}(F) / \operatorname{ker}\left(\left(q_{1}\right)_{*}\right) \longmapsto H \stackrel{j}{\rightarrow} Q$. 
Proposition 4.1. As a subring of the quaternionic integers,

$$
H_{1}(F) / \operatorname{ker}\left(\left(q_{1}\right)_{*}\right) \cong \operatorname{ker}(\psi),
$$

where $\psi: \mathbb{Z}[1, i, j, k] \rightarrow \mathbb{Z}_{2}$ is given by summing coordinates. Explicitly, we can say $\operatorname{ker}(\psi)=\mathbb{Z}[2,1+i, i-j, j+k]$. Thus our extension is

$$
\mathbb{Z}[2,1+i, i-j, j+k] \mapsto H \stackrel{j}{\rightarrow} Q \text { or } \mathbb{Z}^{4} \mapsto H \stackrel{j}{\rightarrow} Q
$$

where the action of $Q$ is by quaternionic multiplication in $\operatorname{ker}(\psi)$.

Proof. We wish to characterize all pairs $(a, b) \in \mathbb{Z}[1, i, j, k] \oplus \mathbb{Z}[1, i, j, k]$ such that

$$
a(i-1)+b(j-1)=0 .
$$

We proceed:

$$
\begin{gathered}
a(i-1)=-b(j-1), \quad a=-b(j-1)(i-1)^{-1}, \\
a=-b(j-1)\left(\frac{i+1}{2}\right), \quad 2 a=-b(j-1)(i+1), \\
2 a=-b(-1-i+j-k) .
\end{gathered}
$$

Let $a=a_{1}+a_{2} i+a_{3} j+a_{4} k$ and $b=b_{1}+b_{2} i+b_{3} j+b_{4} k$. Then we have

$$
\begin{aligned}
2 a= & \left(-b_{1}-b_{2} i-b_{3} j-b_{4} k\right)(-1-i+j-k) \\
2 a= & \left(b_{1}-b_{2}+b_{3}-b_{4}\right)+\left(b_{1}+b_{2}+b_{3}+b_{4}\right) i \\
& \cdot\left(-b_{1}-b_{2}+b_{3}+b_{4}\right) j+\left(b_{1}-b_{2}-b_{3}+b_{4}\right) k .
\end{aligned}
$$

From this description we can conclude

$$
\begin{aligned}
(a, b) \in H_{1}(F) / \operatorname{ker}\left(q_{1}\right)_{*} & \Leftrightarrow 2 a=-b(j-1)(i+1) \quad \text { for some } a \in \mathbb{Z}[1, i, j, k] \\
& \Leftrightarrow \sum b_{i} \equiv 0 \bmod 2 \\
& \Leftrightarrow b \in \operatorname{ker}(\psi) .
\end{aligned}
$$

Since $a$ depends on $b$, we identify $H_{1}(F) / \operatorname{ker}\left(\left(q_{1}\right)_{*}\right)$ with $\operatorname{ker}(\psi)$. In other words we identify the kernel of our extension with the second summand of $\mathbb{Z}[1, i, j, k] \oplus \mathbb{Z}[1, i, j, k]$.

For convenience we denote the generators of $\operatorname{ker}(\psi)$ by $q, r, s, t$.

Proposition 4.2. $\bar{H}$ is given by

$$
\mathbb{Z}_{(2)}[2,1+i, i-j, j+k] \hookrightarrow \bar{H} \stackrel{\bar{j}}{\rightarrow} Q .
$$

Proof. Let $q_{2}: Q \rightarrow \mathbb{Z} \times \mathbb{Z}$ be abelianization. Let $D=\operatorname{ker}\left(j \circ q_{2}\right)$. Then $D$ is given by the extension $\mathbb{Z}[2,1+i, i-j, j+k] \mapsto D \rightarrow \mathbb{Z}_{2}$, action by negation. We write the extension $\mathbb{Z}^{4} \rightarrow D \rightarrow \mathbb{Z}_{2}$. We verify that $D$ is a semidirect product by constructing a splitting, $s$. Let $t$ be the generator of $\mathbb{Z}_{2}$ and define $s(t)=[a, b]$, projected to $D \triangleleft H$. By an explicit lifting argument we show that $[s(t)]^{2}=e \in D$. Since $D \triangleleft H$ we do computations in $F_{2}$ and $H$. Any loop $\gamma$ in $K\left(F_{2}, 1\right)$ representing $[a, b]^{2} \in F_{2}$ and based at the wedge point $x_{0}$ lifts canonically to a 1 -chain in the based cover $\left(K(F, 1), \widetilde{x_{0}}\right)$ by stipulating that $\tilde{\gamma}(0)=\widetilde{x_{0}}$. Algebraically, this chain is an element of $\mathbb{Z}[Q] \oplus \mathbb{Z}[Q]$ and is

$$
\left(1-x y x^{-1}+[x, y]-[x, y] x y x^{-1}\right) \oplus\left(x-[x, y]+[x, y] x-[x, y]^{2}\right) .
$$


The map $\mathbb{Z}[Q] \rightarrow \mathbb{Z}[1, i, j, k]$ induced by $q_{1}$ allows us to write this as a chain in $\mathbb{Z}[1, i, j, k] \oplus \mathbb{Z}[1, i, j, k]$. We obtain

$$
(1+j-1-j) \oplus(i+1-i-1)=0 \oplus 0 .
$$

This is evidently a cycle which represents zero and we conclude that

$$
s(t)^{2}=e \in \operatorname{ker}(\psi) \triangleleft D \triangleleft H .
$$

This means that $s$ is a splitting. We determine $\bar{H}$ by taking the algebraic closure $\hat{D}$ of $D$ in the sense of Levine. The argument of Proposition 2 of [L1] shows that $\hat{D}$ is given by $\mathbb{Z}_{(2)}^{4} \nrightarrow \hat{D} \rightarrow \mathbb{Z}_{2}$. By definition of the $\mathbb{Z}^{n+2}$-algebraic closure, we get the result of the proposition.

We think of $H$ as a quotient of $G$ via the composition $p \circ \mathrm{pr}_{2}$, where we take $\operatorname{pr}_{2}: G \cong \mathbb{Z}^{n} \times F_{2} \rightarrow F_{2}$ to be the natural projection and $p: F_{2} \rightarrow H$ to be the composition of the above quotients.

We seek to discover words in $F_{2}$ that map to generators of $\operatorname{ker}(\psi)$ under the projection $p: F_{2} \rightarrow H$.

Proposition 4.3. The word $\left[a[a, b] a^{-1},[a, b]\right] \in F_{2}$ maps to $q^{2} \in \operatorname{ker}(\psi) \triangleleft H$ under the projection $p: F_{2} \rightarrow H$.

Proof. Again, we locate the word in $H$ by an explicit lifting argument in $F_{2}$ followed by a projection to $H$. We use the free calculus and find that in $\mathbb{Z}[Q] \oplus$ $\mathbb{Z}[Q]$, our word lifts to

$$
\begin{aligned}
(1+x & -x^{2} y x^{-1}-x[x, y] x^{-1}+x[x, y] x^{-1}-x[x, y] x^{-1} x y x^{-1} \\
& +x[x, y] x^{-1}[x, y]+x[x, y] x^{-1}[x, y] x y-x[x, y] x^{-1}[x, y] x[y, x] \\
& -x[x, y] x^{-1}[x, y] x[y, x] x^{-1}+x[x, y] x^{-1}[x, y] x[y, x] x^{-1} y \\
& \left.-x[x, y] x^{-1}[x, y] x[y, x] x^{-1}[y, x]\right) \\
\oplus & \left(x^{2}-x[x, y]+x[x, y] x^{-1} x-x[x, y] x^{-1}[x, y]\right. \\
& +x[x, y] x^{-1}[x, y] x-x[x, y] x^{-1}[x, y] x y x y^{-1} \\
& \left.+x[x, y] x^{-1}[x, y] x[y, x] x^{-1}-x[x, y] x^{-1}[x, y] x[y, x] x^{-1} y x y^{-1}\right) .
\end{aligned}
$$

In $\mathbb{Z}[1, i, j, k] \oplus \mathbb{Z}[1, i, j, k]$, this is

$$
\begin{aligned}
& (1+i+k+1-1-j+1+k+i+1-j-1) \\
& \quad \oplus(-1+i-i-1+i-1-1-i)=(2+2 i-2 j+2 k) \oplus(-4) .
\end{aligned}
$$

We see that this can be considered $q^{2} \in \operatorname{ker}(\psi) \triangleleft H$, because $q$ is our symbol for -2 and the kernel of our extension of $H$ is identified with the second summand of $\mathbb{Z}[1, i, j, k] \oplus \mathbb{Z}[1, i, j, k]$. To conclude we verify that we have found a cycle:

$$
\begin{aligned}
& (2+2 i-2 j+2 k)(i-1)+(-4)(j-1) \\
& \quad=2 i-2-2-2 i+2 k+2 j+2 j-2 k-4 j+4=0 .
\end{aligned}
$$

To perform calculations we will need to consider a certain index four subgroup of $H$. Define $\kappa: Q \rightarrow \mathbb{Z}_{2} \times \mathbb{Z}_{2}$ by letting $\kappa(x)=(1,0)$ and $\kappa(y)=$ $(0,1)$. Let $K \triangleleft H$ be determined by the composite $\kappa \circ j: H \rightarrow \mathbb{Z}_{2} \times \mathbb{Z}_{2}$. 
Proposition 4.4. $\widetilde{Q} \stackrel{\text { def }}{=} \operatorname{ker}(\kappa) \cong \mathbb{Z} \times \mathbb{Z} \times \mathbb{Z}_{2}$. Thus, $K$ is given by the extension $\mathbb{Z}^{4} \mapsto K \stackrel{j}{\rightarrow} \mathbb{Z} \times \mathbb{Z} \times \mathbb{Z}_{2}$ where the action of all generators is by negation. Indeed, we can specify $K$ explicitly with generators and relations:

$$
\begin{array}{r}
K \cong\langle q, r, s, t, u, v, w|[u, v]=r^{-2} s^{2},[u, w]=r^{-2},[v, w]=s^{-2} \\
\left.w^{2}=e,[q, r]=[q, s]=[q, t]=[r, s]=[r, t]=[s, t]=e\right\rangle .
\end{array}
$$

Similarly, we define $\bar{K} \triangleleft \bar{H}$, the $\overline{\mathscr{H}}$-localization or $\mathbb{Z}^{n+2}$-algebraic closure of $K$. It is given by the extension $\mathbb{Z}_{(2)}^{4} \mapsto \bar{K} \stackrel{\bar{j}}{\rightarrow} \mathbb{Z} \times \mathbb{Z} \times \mathbb{Z}_{2}$.

Proof. We have the following group diagram:

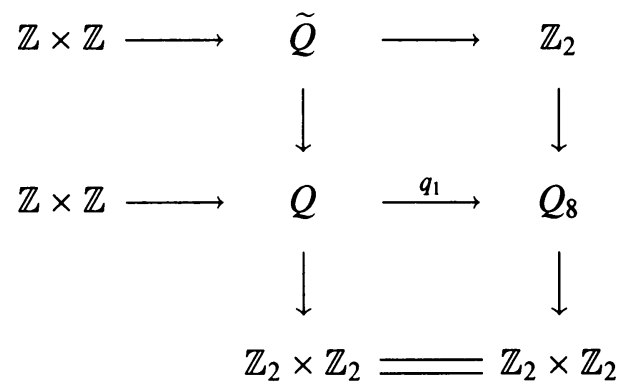

where $Q_{8}$ is the group of quaternionic units. In fact, since $x^{2}, y^{2}$, and $[x, y]$ are in $\operatorname{ker}(\kappa)$, we have the following description of $\widetilde{Q}$ :

(*) $\left\langle x^{2}, y^{2},[x, y] \mid[x, y]^{2}=\left[x^{2},[x, y]\right]=\left[y^{2},[x, y]\right]=\left[x^{2}, y^{2}\right]=e\right\rangle$.

The first two relations are inherited from $Q$. Since $x y=[x, y] y x$ in $Q$, we have

$$
\begin{aligned}
x^{2} y^{2} x^{-2} y^{-2} & =x y x[x, y] y x^{-2} y^{-2}=x y[x, y]^{2} y x^{-1} y^{-2} \\
& =y x[x, y] y x^{-1} y^{-2}=y[x, y]^{2} y x x^{-1} y^{-2}=e .
\end{aligned}
$$

Of course, $(*)$ is just the statement that

$$
\widetilde{Q} \cong \mathbb{Z} \times \mathbb{Z} \times \mathbb{Z}_{2}
$$

And we have

$$
\mathbb{Z}^{4} \longmapsto H \rightarrow \mathbb{Z} \times \mathbb{Z} \times \mathbb{Z}_{2}, \quad \mathbb{Z}_{(2)}^{4} \hookleftarrow \bar{H} \rightarrow \mathbb{Z} \times \mathbb{Z} \times \mathbb{Z}_{2}
$$

action all by negation. The localization is computed as in Proposition 4.2. We work out the presentation of $K$ by explicit lifting arguments in $H \triangleright K$. We already verified, in the proof of Proposition 4.2 , that $[x, y]^{2}=e$. For $\left[x^{2},[x, y]\right]$, we find that in $\mathbb{Z}[Q] \oplus \mathbb{Z}[Q]$ we have

$$
\begin{aligned}
& \left(1+x+x^{2}-x^{3} y x^{-1}-x^{2}[x, y] x^{-1}\right. \\
& \left.\quad-x^{2}[x, y] x^{-2}+x^{2}[x, y] x^{-2} y-x^{2}[x, y] x^{-2}[y, x]\right) \\
& \quad \oplus\left(x^{3}-x^{2}[x, y]+x^{2}[x, y] x^{-2}-x^{2}[x, y] x^{-2} y x y^{-1}\right) .
\end{aligned}
$$

In $\mathbb{Z}[1, i, j, k] \oplus \mathbb{Z}[1, i, j, k]$, this is

$$
(1+i-1-j+i+1-j-1) \oplus(-i-1-1-i)=(2 i-2 j) \oplus(-2-2 i) \text {. }
$$


This is $r^{-2}$ in $\operatorname{ker}(\psi)$. We verify this is a cycle:

$$
\begin{aligned}
& (2 i-2 j)(i-1)+(-2-2 i)(j-1) \\
& \quad=-2-2 i+2 k+2 j-2 j+2-2 k+2 i=0 .
\end{aligned}
$$

For $\left[y^{2},[x, y]\right]$, we find that in $\mathbb{Z}[Q] \oplus \mathbb{Z}[Q]$ we have

$$
\begin{aligned}
& \left(y^{2}-y^{2} x y x^{-1}+y^{2}[x, y] y^{-1}-y^{2}[x, y] y^{-2}[y, x]\right) \\
& \quad \oplus\left(1+y+y^{2} x-y^{2}[x, y]-y^{2}[x, y] y^{-1}-y^{2}[x, y] y^{-1} x y^{-1}\right) .
\end{aligned}
$$

In $\mathbb{Z}[1, i, j, k] \oplus \mathbb{Z}[1, i, j, k]$, this is

$$
(-1-j-j-1) \oplus(1+j-i-1+j-i)=(-2-2 j) \oplus(-2 i+2 j) .
$$

This is $s^{-2}$ in $\operatorname{ker}(\psi)$. We verify that this is a cycle:

$$
\begin{aligned}
& (-2-2 j)(i-1)+(-2 i+2 j)(j-1) \\
& \quad=-2 i+2+2 k+2 j-2 k+2 i-2-2 j=0 .
\end{aligned}
$$

For $\left[x^{2}, y^{2}\right]$, we find that in $\mathbb{Z}[Q] \oplus \mathbb{Z}[Q]$ we have

$$
\left(1+x-x^{2} y^{2} x^{-1}-x^{2} y^{2} x^{-2}\right) \oplus\left(x^{2}+x^{2} y-x^{2} y^{2} x^{-2} y^{-1}-x^{2} y^{2} x^{-2} y^{-2}\right) .
$$

In $\mathbb{Z}[1, i, j, k] \oplus \mathbb{Z}[1, i, j, k]$, this is

$$
\begin{gathered}
(1+i+i+1) \oplus(-1-j-j-1), \\
(2+2 i) \oplus(-2-2 j) .
\end{gathered}
$$

Since $-2-2 j=-2(1+i-(i-j))$, we see that in $\operatorname{ker}(\psi)$ we have $r^{-2} s^{2}$. We verify that we have a cycle:

$$
\begin{aligned}
& (2+2 i)(i-1)+(-2-2 j)(j-1) \\
& \quad=2 i-2-2-2 i-2 j+2+2+2 j=0 .
\end{aligned}
$$

Denoting $x^{2}$ by $u, y^{2}$ by $v$, and $[x, y]$ by $w$, we get the result.

If $z \in \mathbb{Z}_{(2)}^{4}$, we write it as $z_{1} q+z_{2} r+z_{3} s+z_{4} t$ where $z_{i} \in \mathbb{Z}_{(2)}$. The homomorphism $\kappa$ also determines index four subgroups $J \triangleleft G$ and $\bar{J} \triangleleft \bar{G}$ via the composition $\kappa \circ \varphi \circ \mathrm{pr}_{2}: G \rightarrow \mathbb{Z}_{2} \times \mathbb{Z}_{2}$.

\section{COBORDISM INVARIANTS}

In [APS], an invariant $\rho_{\alpha}(M)$ is defined for an oriented manifold with boundary, $M$, of odd dimension equipped with a unitary representation of its fundamental group, $\alpha: \pi_{1}(M) \rightarrow U(k)$. In general, the $\rho$-invariant is a real number, and is defined in terms of the spectrum of a certain differential operator. But if $M=\partial V$ and $\alpha$ factors through a representation $\alpha^{\prime}: \pi_{1}(V) \rightarrow U(k)$, then the $\rho$-invariant has the following alternate description:

$$
\rho_{\alpha}(M)=k \sigma(V)-\sigma_{\alpha^{\prime}}(V)
$$

where $\sigma$ is the ordinary signature and $\sigma_{\alpha^{\prime}}$ is the signature using local coefficients determined by $\alpha^{\prime}$.

We adapt the approach of Levine [L3] to the category $\mathscr{G}$. Let $(M, \alpha)$ be a $G$-manifold equipped with $\mathbb{Z}\left[\mathbb{Z}^{n+2}\right]$ coefficients, where $\alpha$ is a morphism in $\mathscr{G}$. This means, in particular, that $\alpha: \pi_{1}(M) \rightarrow G$ is a group homomorphism. 
Let $R_{k}(G)$ denote the set of unitary representations $G \rightarrow U(k)$. We construct a signature function

by defining

$$
\sigma(M, \alpha): R_{k}(G) \rightarrow \mathbb{R}
$$

where $\theta \in R_{k}(G)$.

$$
\sigma(M, \alpha) \bullet \theta=\rho_{\theta \alpha}(M)
$$

Suppose $\left(\lambda_{i j}\right)$ is a square matrix with entries in $\mathbb{Z}[G]$ such that the matrix $\left(\mathscr{F}\left(\lambda_{i j}\right)\right)$ over $\mathbb{Z}\left[\mathbb{Z}^{n+2}\right]$, where $\mathscr{F}$ is induced by the $\mathbb{Z}^{n+2}$-structure of $G$, is nonsingular. Define

$$
g: R_{k}(G) \rightarrow \mathbb{Z}, \quad g(\theta)=\left|\operatorname{det}\left(\theta\left(\lambda_{i j}\right)\right)\right|^{2} .
$$

Any such function will be called $\mathbb{Z}^{n+2}$-special. The complement of the zero set of a special function will be called large.

Proposition 5.1. If $(M, \alpha)$ and $\left(M^{\prime}, \alpha^{\prime}\right)$ are G-manifolds equipped with local coefficient systems over $\mathbb{Z}\left[\mathbb{Z}^{n+2}\right], \alpha$ and $\alpha^{\prime}$ are morphisms in $\mathscr{G}$, the two manifolds are $\mathbb{Z}\left[\mathbb{Z}^{n+2}\right]$-homology $G$-bordant, and $M=\partial V$ where $(V, \beta)$ is a $G$-manifold compatible with the inclusion of $(M, \alpha)$, then

$$
\sigma(M, \alpha)=\sigma\left(M^{\prime}, \alpha^{\prime}\right)
$$

on some large subset of $R_{k}(G)$.

Proof. Let $(W, \gamma)$ be the $\mathbb{Z}\left[\mathbb{Z}^{n+2}\right]$-homology $G$-bordism between $(M, \alpha)$ and $\left(M^{\prime}, \alpha^{\prime}\right)$, i.e., $\gamma: \pi_{1} W \rightarrow G$ is a morphsim in $\mathscr{G}$. Let $\left(\lambda_{i j}\right)$ be the intersection form on homology of $V$ with local coefficients in $\mathbb{Z}\left[\pi_{1}(V)\right]$. Let

$$
g: R_{k}(G) \rightarrow \mathbb{Z}, \quad g(\theta)=\left|\operatorname{det}\left(\theta \beta\left(\lambda_{i j}\right)\right)\right|^{2} .
$$

Let $U$ be the complement of the zero set of $g$. Then for all $\theta \in U$ we have

$$
\rho_{\theta \alpha}(M)=k \sigma(V)-\sigma_{\theta \beta}(V) .
$$

Since $H_{*}\left(W, M ; \mathbb{Z}\left[\mathbb{Z}^{n+2}\right]\right)=0$,

$$
\sigma_{\theta \gamma}(W)=\sigma_{\theta \alpha}(M)=0, \quad \sigma(W)=\sigma(M)=0 .
$$

Now $M^{\prime}=\partial\left(V \cup_{M} W\right)$, so the additivity of the ordinary signature and of the signature with local coefficients implies that

$$
\rho_{\theta \alpha}(M)=\rho_{\theta \alpha^{\prime}}\left(M^{\prime}\right)
$$

for all $\theta$ with $g(\theta) \neq 0$. Thus,

$$
\sigma(M, \alpha)=\sigma\left(M^{\prime}, \alpha^{\prime}\right)
$$

on a large subset of $R_{k}(G)$.

Definition 5.1. If $f: T^{n} \rightarrow T^{n+2}$ is a $G$-embedding equipped with $G$-structure $\alpha$, then

$$
\sigma_{G}(f) \stackrel{\text { def }}{=} \sigma(M(f), \alpha) .
$$

Proposition 5.2. If $f_{0}, f_{1}: T^{n} \rightarrow T^{n+2}$ are G-cobordant G-embeddings, then

$$
\sigma_{G}\left(f_{0}\right)=\sigma_{G}\left(f_{1}\right)
$$

on some large subset of $R_{k}(G)$. 
Proof. This is a result of Propositions 1.1 and 5.1.

Let $\mathscr{F}: \mathbb{Z}[G] \rightarrow \Lambda$ be a ring homomorphism compatible with involution. There are algebraically defined abelian groups $\Gamma_{n+3}(\mathscr{F})$ associated to $\mathscr{F}$ for $n$ odd [CS1]. These groups are equivalence classes of triples $(B, \lambda, \mu)$ where $B$ is a finitely generated module over $\mathbb{Z}[G], \lambda$ is a bilinear form over $B$, and $\mu: B \rightarrow \mathbb{Z}[G] /\left\{\gamma-(-1)^{(n+3) / 2} \bar{\gamma} \mid \gamma \in \mathbb{Z}[G]\right\}$ is a quadratic form, and $\lambda, \mu$ satisfy $(\mathrm{Q} 1)-(\mathrm{Q} 6)$ of [CS1]. Specifically, $B \otimes_{\mathbb{Z}[G]} \Lambda$ is stably based and the form induced from $\lambda$ is nonsingular. Two triples $(B, \lambda, \mu)$, and $\left(B^{\prime}, \lambda^{\prime}, \mu^{\prime}\right)$ are equivalent if the orthogonal direct sum

$$
(B, \lambda, \mu) \perp\left(B^{\prime},-\lambda^{\prime},-\mu^{\prime}\right) \approx 0
$$

where $\approx 0$ denotes strong equivalence to zero in the sense of [CS1].

If $\lambda$ is the bilinear form for the triple $(B, \lambda, \mu)$ and $\theta \in R_{k}(G)$, then one obtains an associated complex Hermitian form $\lambda_{\theta}$ on $\mathbb{C} \otimes_{\theta} B$. Define $\sigma(B, \lambda, \mu): R_{k}(G) \rightarrow \mathbb{R}$ by

$$
\sigma(B, \lambda, \mu) \bullet \theta=\text { signature } \lambda_{\theta} .
$$

Proposition 5.3. If $(B, \lambda, \mu)$ and $\left(B^{\prime}, \lambda^{\prime}, \mu^{\prime}\right)$ represent the same element of $\Gamma_{n+3}(\mathscr{F})$, then

$$
\sigma(B, \lambda, \mu)=\sigma\left(B^{\prime}, \lambda^{\prime}, \mu^{\prime}\right)
$$

on a large subset of $R_{k}(G)$.

Proof. Define

$$
\begin{aligned}
& \Delta(B, \lambda, \mu): R_{k}(G) \rightarrow \mathbb{Z}, \\
& \Delta(B, \lambda, \mu) \bullet \theta=\left|\operatorname{det}\left(\lambda_{\theta}\right)\right|^{2} .
\end{aligned}
$$

Let $V(B, \lambda, \mu)$ be the complement of the zero set of $\Delta(B, \lambda, \mu)$. Then $\Delta(B, \lambda, \mu)$ is $\mathbb{Z}^{n+2}$-special and $V(B, \lambda, \mu)$ is large. Recall that

$$
\left(B \oplus B^{\prime}, \lambda \oplus\left(-\lambda^{\prime}\right), \mu \oplus\left(-\mu^{\prime}\right)\right) \approx 0 .
$$

By definition of strong equivalence to zero, there exists a submodule $K \subset B \oplus B^{\prime}$ of half rank such that

$$
\left(\lambda \oplus\left(-\lambda^{\prime}\right)\right)(K \times K)=0 .
$$

This means that if $\theta \in V(B, \lambda, \mu)$, then

$$
\left(\lambda_{\theta} \oplus\left(-\lambda^{\prime}\right)_{\theta}\right)\left(K \otimes_{\theta} \mathbb{C} \times K \otimes_{\theta} \mathbb{C}\right)=0 .
$$

Since $\lambda_{\theta} \oplus\left(-\lambda^{\prime}\right)_{\theta}$ is zero on a submodule of half rank, we know that

$$
\text { signature }\left(\lambda_{\theta} \oplus\left(-\lambda^{\prime}\right)_{\theta}\right)=0 .
$$

By additivity of the signature this implies that

$$
\sigma(B, \lambda, \mu) \bullet \theta=\sigma\left(B^{\prime}, \lambda^{\prime}, \mu^{\prime}\right) \bullet \theta
$$

for all $\theta \in V(B, \lambda, \mu)$. 
Recall the subgroup $K \triangleleft H$ of $\S 4$, and its presentation with generators and relations. We define a map

$$
\begin{aligned}
\phi: \mathbb{R} & \rightarrow R_{2}(K), \\
\phi(x) \bullet u & =\phi(x) \bullet v=\phi(x) \bullet w=\left(\begin{array}{ll}
0 & 1 \\
1 & 0
\end{array}\right), \\
\phi(x) \bullet q & =\phi(x) \bullet r=\phi(x) \bullet s=\left(\begin{array}{ll}
1 & 0 \\
0 & 1
\end{array}\right), \\
\phi(x) \bullet t & =\left(\begin{array}{cc}
e^{2 \pi i x} & 0 \\
0 & e^{-2 \pi i x}
\end{array}\right) .
\end{aligned}
$$

Clearly, $\phi$ has period 1. Recall the localization $\bar{K}$ of $K$. We define $\bar{\phi}: \mathbb{R} \rightarrow$ $R_{2}(\bar{K})$ similarly:

$$
\begin{aligned}
\bar{\phi}(x) \bullet u & =\bar{\phi}(x) \bullet v=\bar{\phi}(x) \bullet w=\left(\begin{array}{ll}
0 & 1 \\
1 & 0
\end{array}\right), \\
\bar{\phi}(x) \bullet(z q) & =\bar{\phi}(x) \bullet(z r)=\bar{\phi}(x) \bullet(z s)=\left(\begin{array}{ll}
1 & 0 \\
0 & 1
\end{array}\right), \\
\bar{\phi}(x) \bullet(z t) & =\left(\begin{array}{cc}
e^{2 \pi i x z} & 0 \\
0 & e^{-2 \pi i x z}
\end{array}\right)
\end{aligned}
$$

for any $z \in \mathbb{Z}_{(2)}$. Note that $\left.\bar{\phi}(x)\right|_{K}=\phi(x): K \rightarrow U(2)$ for all $x \in \mathbb{R}$. Thus if $(M, \alpha)$ is a $K$-manifold, or respectively $(M, \bar{\alpha})$ is a $\bar{K}$-manifold, we can consider the compositions

$$
\sigma(M, \alpha) \circ \phi: \mathbb{R} \rightarrow \mathbb{R}, \quad \bar{\sigma}(M, \bar{\alpha}) \circ \bar{\phi}: \mathbb{R} \rightarrow \mathbb{R} .
$$

The former composition necessarily has period 1 . Now if $\ell: K \rightarrow \bar{K}$ is the inclusion in the localization, we have the map $l^{*}: R_{2}(\bar{K}) \rightarrow R_{2}(K)$, and $\ell^{*} \bar{\phi}: \mathbb{R} \rightarrow R_{2}(K)$ has period 1. Thus if $(M, \bar{\alpha})$ is a $\bar{K}$-manifold, and $\bar{\sigma}(M, \bar{\alpha}) \circ \bar{\phi}$ does not have period 1 , then $\bar{\alpha}\left(\pi_{1} M\right)$ is not contained in $l(K)$. Moreover, by Proposition 5.1, if $(M, \bar{\alpha})$ and $\left(M^{\prime}, \bar{\alpha}^{\prime}\right)$ are $\mathbb{Z}\left[\mathbb{Z}^{n+2}\right]$-homology cobordant $\bar{K}$-manifolds and $\bar{\sigma}(M, \bar{\alpha}) \circ \bar{\phi}$ does not have period 1 , then neither does $\bar{\sigma}\left(M^{\prime}, \bar{\alpha}^{\prime}\right) \circ \bar{\phi}$.

\section{NONCHARACTERISTIC EMBEDDINGS}

In the concluding remarks of [CS2], a program for studying the general link cobordism problem is outlined. This program has been succesfully implemented in [CO2] to discover noncharacteristic links. In this section, the machinery we have developed will allow us to construct noncharacteristic embeddings of tori in an analogous fashion: build a universal object with respect to fundamental groups of complements and perform geometric realization on elements of the Cappell-Shaneson $\Gamma$-group associated to this object to obtain examples. Throughout this section, we let $X, G$, and $H$ be as in $\S \S 1$ and 4 and we let $\mathscr{F}: \mathbb{Z}[G] \rightarrow \mathbb{Z}\left[\mathbb{Z}^{n+2}\right]$ and $\bar{F}: \mathbb{Z}[\bar{G}] \rightarrow \mathbb{Z}\left[\mathbb{Z}^{n+2}\right]$ be induced by abelianization. Define involutions on $\mathbb{Z}[G]$ and $\mathbb{Z}[\bar{G}]$ via the orientation characters on $X$ and $\bar{X}$. For $n=4 k+1, n \geq 5$, we specify classes $\gamma_{n}=[(H, \phi, \mu)] \in \Gamma_{n+3}(\overline{\mathscr{F}})$. First we consider the following intersection form:

$$
\left(\begin{array}{cc}
2\left(a b+(a b)^{-1}-b a-(b a)^{-1}\right)+2 & 3 \\
3 & 4\left(a b+(a b)^{-1}-b a-(b a)^{-1}\right)+4
\end{array}\right),
$$


where $a, b$ are, as before, the generators of the free factor of $G$. The $\mu$ form is given by $2(a b-b a)+1$ and $4(a b-b a)+2$ on generators. Next we define a group homomorphism $g: G \rightarrow \bar{G}$ by stipulating that $g\left(t_{i}\right)=s_{i}$, $i=1, \ldots, n, g\left(c^{k} a\right)=u, g(b)=v$, where $c$ is the word $\left[a[a, b] a^{-1},[a, b]\right]$ and $s_{i}, u, v$ denote the standard generators of $\bar{G}$. The word $c$ is zero as an element of $H_{1}\left(G ; \mathbb{Z}\left[\mathbb{Z}^{n+2}\right]\right)$. Since $\bar{G}$ is a $\mathbb{Z}^{n+2}$-algebraic closure this means that the system

$$
u=g\left(c^{k}\right) g(a), \quad v=g(b)
$$

can be solved for $g(a)$ and $g(b)$. Hence $g$ is a homomorphism with $g(a)=$ $z u$ for some word $g \in \bar{G}$. In fact $g$ is a morphism in $\mathscr{G}$. We let $\gamma_{n}$ denote the class with the intersection form obtained by applying $\bigotimes_{g} \mathbb{Z}[\bar{G}]$ to the above form. Under the natural surjection induced by the group abelianization $h$, $\Gamma_{n+3}(\overline{\mathscr{F}}) \stackrel{h_{*}}{\rightarrow} L_{n+3}\left(\mathbb{Z}^{n+2}\right), \quad h_{*}\left(\gamma_{n}\right)$ is the class represented by the nonsingular form

$$
\left(\begin{array}{ll}
2 & 3 \\
3 & 4
\end{array}\right)
$$

over $\mathbb{Z}\left[\mathbb{Z}^{n+2}\right]$. We apply the geometric realization theorem for $\Gamma$-groups [CS1] to $\gamma_{n}$ to obtain a cobordism $(W, \bar{X}, M)$ and a map $F:(W, \bar{X}, M) \rightarrow(\bar{X} \times$ $I, \bar{X} \times 0, \bar{X} \times 1)$ where $\left.F\right|_{\bar{X}}=\left.\mathrm{id}\right|_{\bar{X}},\left.F\right|_{M}$ is a $\mathbb{Z}\left[\mathbb{Z}^{n+2}\right]$-homology equivalence, and $\sigma(W)=\gamma_{n}$.

Lemma 6.1. $M$ is the closed complement of a tubular neighborhood of an embedding $f: T^{n} \rightarrow T^{n+2}$.

Proof. Consider the space $N$ that makes the following diagram commute:

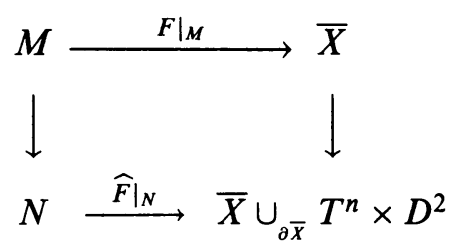

By construction of $\bar{X}, \bar{X} \cup_{\partial \bar{X}} T^{n} \times D^{2}$ is homotopy equivalent to $T^{n+2}$. Hence, we have a cobordism

$$
\left(\widehat{W}, T^{n+2}, N\right) \stackrel{\widehat{F}}{\rightarrow}\left(T^{n+2} \times I, T^{n+2} \times 0, T^{n+2} \times 1\right)
$$

with the surgery obstruction $\sigma(\widehat{W})=h_{*}\left(\gamma_{n}\right)$, and $\left.\widehat{F}\right|_{N}$ a homotopy equivalence: i.e., $\widehat{W}$ is the Wall realization of $h_{*}\left(\gamma_{n}\right)$. But as $h_{*}\left(\gamma_{n}\right)$ is an element of $L_{n+3}\left(\mathbb{Z}^{n+2}\right)$ which gives a standard torus upon realization [HS], $\left.\widehat{F}\right|_{N}$ is homotopic to a homeomorphism with $T^{n+2}$. Since $\left.\widehat{F}\right|_{\partial M}: \partial M \rightarrow \partial \bar{X}=T^{n} \times S^{1}$ is a homeomorphism, the result follows.

Theorem. There exist noncharacteristic embeddings of $T^{n}$ in $T^{n+2}$ that are not cobordant to characteristic embeddings. The map $f: T^{n} \rightarrow T^{n+2}$ of Lemma 6.1 is such an example. 
Proof of Theorem. To study the form $\gamma_{n}$ we must understand the homomorphism $g$. We consider the following commutative diagram:

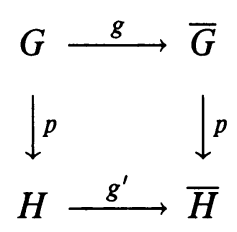

Proposition 4.3 tells us that under $p$, the equation

$$
g\left(c^{k} a\right)=u
$$

gives the equation

$$
g^{\prime}\left(q^{2 k} x\right)=x^{\prime}
$$

where $x \stackrel{\text { def }}{=} p(a)$ and $x^{\prime} \stackrel{\text { def }}{=} p(u)$. This equation determines $\bar{z}=p(z) \epsilon$ $\mathbb{Z}_{(2)}[1, i, j, k]$, as we have

$$
g^{\prime}\left(q^{2 k}\right) g^{\prime}(x)=x^{\prime}, \quad g^{\prime}\left(q^{2}\right)^{k} \bar{z} x^{\prime}=x^{\prime} .
$$

We simply compute $g^{\prime}\left(q^{2}\right)$ directly and cancel $x^{\prime}$ leaving us with a quaternionic equation in $\mathbb{Z}_{(2)}[2,1+i, i-j, j+k]$ which we solve for $\bar{z}$. We know that $\bar{z} \in \mathbb{Z}_{(2)}[2,1+i, i-j, j+k]$ because $g$ is an $\overline{\mathscr{C}}$-map. We find

$$
g^{\prime}\left(q^{2}\right)=g^{\prime}\left(\left[x[x, y] x^{-1},[x, y]\right)=\left[\bar{z} x^{\prime}\left[\bar{z} x^{\prime}, y^{\prime}\right]\left(\bar{z} x^{\prime}\right)^{-1},\left[\bar{z} x^{\prime}, y^{\prime}\right]\right]=z_{*}\left(q^{\prime}\right)^{2},\right.
$$

where $z_{*}$ is obtained from the action of $Q$ in $\bar{H}$, ie, by quaternionic multiplication effected by the relations $x^{\prime} \bar{z}=(\bar{z} \cdot i) x^{\prime}$, and $y^{\prime} \bar{z}=(\bar{z} \cdot j) y^{\prime}$. So

$$
\begin{aligned}
z_{*}= & \bar{z}+\bar{z} \cdot i+(-\bar{z}) \cdot(-i) \cdot j \cdot i^{2}+(-\bar{z}) \cdot(-i)^{2} \cdot j \cdot i^{2} \\
& +\bar{z} \cdot(-j) \cdot(-i)^{2} \cdot j \cdot i^{2}+\bar{z} \cdot j \cdot i \cdot 1+(-\bar{z}) \cdot(-i) \cdot(-j) \cdot i \cdot j \cdot i \cdot 1 \\
& +(-\bar{z}) \cdot(-i)^{2} \cdot(-j) \cdot i \cdot j \cdot i+\bar{z} \cdot j \cdot 1+(-\bar{z}) \cdot(-i) \cdot(-j) \cdot i \cdot j \cdot 1, \\
z_{*}= & \bar{z}+\bar{z} \cdot i+(-\bar{z}) \cdot k+(-\bar{z}) \cdot j+\bar{z}+(-\bar{z}) \cdot k+\bar{z} \cdot i+\bar{z}+\bar{z} \cdot j+\bar{z}, \\
z_{*}= & 4 \bar{z}+2 \bar{z} \cdot i-2 \bar{z} \cdot k .
\end{aligned}
$$

Thus our equation becomes

$$
\left((\bar{z} \cdot(4+2 \vec{i}-2 \vec{k}))\left(q^{\prime}\right)^{2}\right)^{k} \bar{z} x^{\prime}=x^{\prime} .
$$

We write

$$
\bar{z}=z_{1} q^{\prime}+z_{2} r^{\prime}+z_{3} s^{\prime}+z_{4} t^{\prime} .
$$

Cancelling $x^{\prime}$ and interpreting $\mathbb{Z}_{(2)}[2,1+i, i-j, j+k]$ as a subring of $\mathbb{Z}_{(2)}[1, i, j, k]$ we have

$$
k(\bar{z} \cdot(4+2 \vec{i}-2 \vec{k}))+2 k q^{\prime}+\bar{z}=0
$$

or

$$
(4 k+1) \bar{z}+2 k(\bar{z} \cdot \vec{\imath})-2 k(\bar{z} \cdot \vec{k})+2 k q^{\prime}=0
$$


To determine $\bar{z}$ we note that

$$
\begin{aligned}
\bar{z} \cdot i= & \left(z_{1} q^{\prime}+z_{2} r^{\prime}+z_{3} s^{\prime}+z_{4} t^{\prime}\right) i \\
= & \left(2 z_{1}+z_{2}(1+i)+z_{3}(i-j)+z_{4}(j+k)\right) i \\
= & z_{1}(2 i)+z_{2}(-1+i)+z_{3}(-1+k)+z_{4}(j-k) \\
= & z_{1}\left(-q^{\prime}+2 r^{\prime}\right)+z_{2}\left(r^{\prime}-q^{\prime}\right)+z_{3}\left(-r^{\prime}+s^{\prime}+t^{\prime}\right)+z_{4}\left(-q^{\prime}+2 r^{\prime}-2 s^{\prime}-t^{\prime}\right) \\
= & \left(-z_{1}-z_{2}-z_{4}\right) q^{\prime}+\left(2 z_{1}+z_{2}-z_{3}+2 z_{4}\right) r^{\prime}+\left(z_{3}-2 z_{4}\right) s^{\prime}+\left(z_{3}-z_{4}\right) t^{\prime} \\
\bar{z} \cdot k= & \left(z_{1} q^{\prime}+z_{2} r^{\prime}+z_{3} s^{\prime}+z_{4} t^{\prime}\right) k \\
= & \left(2 z_{1}+z_{2}(1+i)+z_{3}(i-j)+z_{4}(j+k)\right) k \\
= & z_{1}(2 k)+z_{2}(k-j)+z_{3}(-i-j)+z_{4}(-1+i) \\
= & z_{1}\left(q^{\prime}-2 r^{\prime}+2 s^{\prime}+2 t^{\prime}\right)+z_{2}\left(q^{\prime}-2 r^{\prime}+2 s^{\prime}+t^{\prime}\right) \\
& +z_{3}\left(q^{\prime}-2 r^{\prime}+s^{\prime}\right)+z_{4}\left(r^{\prime}-q^{\prime}\right) \\
= & \left(z_{1}+z_{2}+z_{3}-z_{4}\right) q^{\prime}+\left(-2 z_{1}-2 z_{2}-2 z_{3}+z_{4}\right) r^{\prime} \\
& +\left(2 z_{1}+2 z_{2}+z_{3}\right) s^{\prime}+\left(2 z_{1}+z_{2}\right) t^{\prime}
\end{aligned}
$$

These two facts allow us to turn $(*)$ into a system of four equations:

$$
\begin{aligned}
0= & (4 k+1) z_{1} q^{\prime}-2 k z_{1} q^{\prime}-2 k z_{2} q^{\prime}-2 k z_{4} q^{\prime}-2 k z_{1} q^{\prime} \\
& -2 k z_{2} q^{\prime}-2 k z_{3} q^{\prime}+2 k z_{4} q^{\prime}+2 k q^{\prime}, \\
0= & (4 k+1) z_{2} r^{\prime}+4 k z_{1} r^{\prime}+2 k z_{2} r^{\prime}-2 k z_{3} r^{\prime}+4 k z_{4} r^{\prime} \\
& +4 k z_{1} r^{\prime}+4 k z_{2} r^{\prime}+4 k z_{3} r^{\prime}-2 k z_{4} r^{\prime}, \\
0= & (4 k+1) z_{3} s^{\prime}+2 k z_{3} s^{\prime}-4 k z_{4} s^{\prime}-4 k z_{1} s^{\prime}-4 k z_{2} s^{\prime}-2 k z_{3} s^{\prime}, \\
0= & (4 k+1) z_{4} t^{\prime}+2 k z_{3} t^{\prime}-2 k z_{4} t^{\prime}-4 k z_{1} t^{\prime}-2 k z_{2} t^{\prime} .
\end{aligned}
$$

Thus equation $(*)$ is equivalent to the system:

$$
\left(\begin{array}{cccc}
1 & -4 k & -2 k & 0 \\
8 k & 10 k+1 & 2 k & 2 k \\
-4 k & -4 k & 2 k+1 & -4 k \\
-4 k & -2 k & 2 k & 2 k+1
\end{array}\right)\left(\begin{array}{c}
z_{1} \\
z_{2} \\
z_{3} \\
z_{4}
\end{array}\right)=\left(\begin{array}{c}
-2 k \\
0 \\
0 \\
0
\end{array}\right) .
$$

The $4 \times 4$-matrix is nonsingular for all $k$, so the system has a unique solution.

For $k=1$, we have the system

$$
\left(\begin{array}{cccc}
1 & -4 & -2 & 0 \\
8 & 11 & 2 & 2 \\
-4 & -4 & 3 & -4 \\
-4 & -2 & 2 & 3
\end{array}\right)\left(\begin{array}{c}
z_{1} \\
z_{2} \\
z_{3} \\
z_{4}
\end{array}\right)=\left(\begin{array}{c}
-2 \\
0 \\
0 \\
0
\end{array}\right)
$$

This matrix is nonsingular, and solving the system we find that

$$
\bar{z}=\left(\frac{-446}{759}\right) q^{\prime}+\left(\frac{400}{759}\right) r^{\prime}+\left(\frac{-264}{759}\right) s^{\prime}+\left(\frac{-152}{759}\right) t^{\prime} .
$$

In order to construct a cobordism invariant, we must pass to the 4-fold cover associated to $(W, X, M)$ by the homomorphism $\kappa: Q \rightarrow \mathbb{Z}_{2} \times \mathbb{Z}_{2}$ described in $\S 5$. In other words, we have the composition $\kappa \circ \jmath \circ p \circ F_{*}: \pi_{1} W \rightarrow \mathbb{Z}_{2} \times \mathbb{Z}_{2}$, and an associated cover $(\widetilde{W}, \widetilde{X}, \widetilde{M})$ and map

$$
\widetilde{F}:(\widetilde{W}, \widetilde{X}, \widetilde{M}) \rightarrow(\widetilde{X} \times I, \widetilde{X} \times 0, \tilde{X} \times 1) .
$$


So the manifold $\widetilde{M}$ has a $\bar{J}$-structure, $\widetilde{\alpha}$. The above triple is a realization of

$$
\operatorname{tr}\left(\gamma_{n}\right) \in \Gamma_{n+3}\left(\mathbb{Z}[\bar{J}] \rightarrow \mathbb{Z}\left[\mathbb{Z}^{n+2}\right]\right) .
$$

We will denote $\mathbb{Z}[\bar{J}] \rightarrow \mathbb{Z}\left[\mathbb{Z}^{n+2}\right]$ by $\widetilde{\mathscr{F}}$. We compute the intersection form of $\operatorname{tr}\left(\gamma_{n}\right)$ explicitly by restricting scalars. The intersection form of $\gamma_{n}$ is a form over a rank-2 $\mathbb{Z}[\bar{G}]$-module $B$. The inclusion-induced ring homomorphism $i_{*} \mathbb{Z}[\bar{J}] \rightarrow \mathbb{Z}[\bar{G}]$ gives a $\mathbb{Z}[\bar{J}]$ module $B_{\bar{J}}$ by

$$
r \cdot m=i_{*}(r) m \text { for all } r \in \mathbb{Z}[\bar{J}], m \in B .
$$

This is restriction of scalars. We can rewrite the intersection form of $\gamma_{n}$ as a form over $B_{\bar{J}}$. This is the intersection form for $\operatorname{tr}\left(\gamma_{n}\right)$.

We think of $\mathbb{Z}[G]$ as a rank four module over $\mathbb{Z}[J]$ induced by the cosets of $J$ in $G$ and study the form $\left(a b+b^{-1} a^{-1}-b a-a^{-1} b^{-1}+1\right)$ over a rank-1 $\mathbb{Z}[G]$ module. Restriction of scalars gives us a form over a free rank-4 $\mathbb{Z}[J]$ module $A$. This form is represented by a $4 \times 4$ matrix whose rows are determined by multiplying the above element, $d$, of $\mathbb{Z}[G]$ by $e, a, b, a b$, respectively, and thinking of the result as an element of $A$ :

$$
\begin{aligned}
e d & =\left(a b+b^{-1} a^{-1}-b a-a^{-1} b^{-1}+1\right) \\
& =\left(1+b^{-1} a^{-1} b^{-1} a^{-1}-b a b^{-1} a^{-1}-a^{-1} b^{-1} b^{-1} a^{-1}\right) a b+1, \\
a d & =a\left(a b+b^{-1} a^{-1}-b a-a^{-1} b^{-1}+1\right) \\
& =a^{2} b+a b^{-1} a^{-1}-a b a-b^{-1}+a \\
& =\left(a^{2}+a b^{-1} a^{-1} b^{-1}-a b a b^{-1}-b^{-2}\right) b+1(a), \\
b d & =b\left(a b+b^{-1} a^{-1}-b a-a^{-1} b^{-1}+1\right) \\
& =b a b+a^{-1}-b^{2} a-b a^{-1} b^{-1}+b \\
& =\left(b a b a^{-1}+a^{-2}-b^{2}-b a^{-1} b^{-1} a^{-1}\right) a+1(b), \\
a b d & =a b\left(a b+b^{-1} a^{-1}-b a-a^{-1} b^{-1}+1\right) \\
& =\left(a b a b+1-a b b a-a b a^{-1} b^{-1}\right)(e)+1(a b) .
\end{aligned}
$$

Thus we get the matrix $P$ :

$$
\left(\begin{array}{cccc}
1 & 0 & 0 & 1+b^{-1} a^{-1} b^{-1} a^{-1} \\
0 & 1 & a^{2}+a b^{-1} a^{-1} b^{-1} & -[b, a]-a^{-1} b^{-2} a^{-1} \\
0 & a^{-2}-b^{2} & -a b a b^{-1}-b^{-2} & 0 \\
{ }_{-a b^{2} a-a b a b}+a b a_{b}-1 & 0 & 1 & 0 \\
+b a a^{-1}-b a^{-1} b^{-1} a^{-1} & 0 & 0 & 1
\end{array}\right)
$$

Let

$$
Q=\left(\begin{array}{cc}
2 P & 3 I_{4 \times 4} \\
3 I_{4 \times 4} & 4 P
\end{array}\right)
$$

Then the intersection matrix for $\operatorname{tr}\left(\gamma_{n}\right)$ is $Q \otimes_{g} \mathbb{Z}[\bar{G}]$. We consider

$$
\eta \stackrel{\text { def }}{=}\left(\left.p\right|_{\bar{J}}\right)_{*}\left(\operatorname{tr}\left(\gamma_{n}\right)\right) \in \Gamma_{n+3}\left(\mathbb{Z}[\bar{K}] \rightarrow \mathbb{Z}\left[\mathbb{Z}^{n+2}\right]\right) .
$$


The matrix for its intersection form looks like the matrix $Q$ with $\bar{z} x^{\prime}$ substituted for $a$ and $y^{\prime}$ substituted for $b$. We now have a form over $\mathbb{Z}[\bar{K}]$. The signature function for $\eta$,

$$
\sigma(\eta): R_{2}(\bar{K}) \rightarrow \mathbb{R}
$$

is an invariant of the choice of representative of the class $\gamma_{n} \in \Gamma_{n+3}(\overline{\mathscr{F}})$ on a large subset of $R_{2}(\bar{K}) . \sigma(\eta)$ is also a $\mathbb{Z}\left[\mathbb{Z}^{n+2}\right]$-homology $\bar{G}$-bordism invariant of $\widetilde{M}$ on a large subset of $R_{2}(\bar{K})$ as it is identical to the geometric signature:

$$
\bar{\sigma}\left(\widetilde{M},\left.p\right|_{\bar{J}} \circ \widetilde{\alpha}\right): R_{2}(\bar{K}) \rightarrow \mathbb{R} \text {. }
$$

We note that if the examples $f$ of Lemma 6.1 are characteristic, then the closure of the complement, $M$, is a $G$-manifold. If the $\bar{G}$ structure $\alpha$ described above on $M$ comes from a $G$-structure, then the $\bar{J}$ structure $\widetilde{\alpha}$ on $\widetilde{M}$ comes from a $J$-structure. This would in turn imply that $\bar{\sigma}\left(\widetilde{M},\left.p\right|_{\bar{J}} \circ \widetilde{\alpha}\right) \circ \bar{\phi}: \mathbb{R} \rightarrow \mathbb{R}$ would have period 1 , where $\bar{\sigma}$ is the signature on $\bar{K}$ as in $\S 5$. We verify that this is not the case by computing $\sigma(\eta) \bullet \bar{\phi}(0)$ and $\sigma(\eta) \bullet \bar{\phi}(1)$ and observing that they differ. $\bar{\phi}(0)$ and $\bar{\phi}(1)$ are in the large subset of $\mathbb{Z}^{n+2}$-homology $\bar{G}$-bordism invariant representations of $R_{2}(\bar{K})$ with respect to the $\rho$-invariant because, if $\widetilde{M}^{\prime}$ is $\mathbb{Z}^{n+2}$-homology $\bar{G}$-bordant to $\widetilde{M}$ via a bordism $\widetilde{W}^{\prime}$, then the surgery obstruction $\sigma\left(\widetilde{W} \cup \widetilde{W}^{\prime}\right)=\sigma(\widetilde{W})$ and Proposition 5.3 confirms that the signatures of $M$ and $\widetilde{M}$ are the same at $\bar{\phi}(0)$ and $\bar{\phi}(1)$. Recall the definition

$$
\sigma(\eta) \bullet \theta=\operatorname{signature}\left(\eta_{\theta}\right) \text {. }
$$

To represent the entries of the intersection form of $\eta$, we simplify the words that appear there as follows:

$$
\begin{aligned}
& b^{-1} a^{-1} b^{-1} a^{-1} \mapsto y^{\prime-1} x^{\prime-1} \bar{z}^{-1} y^{\prime-1} x^{\prime-1} \bar{z}^{-1} \\
&=((-\bar{z}) \cdot(-i) \cdot(-j)) \\
&((-\bar{z}) \cdot(-i) \cdot(-j) \cdot(-i) \cdot(-j)) y^{\prime-1} x^{\prime-1} y^{\prime-1} x^{\prime-1} \\
&= \bar{z}((-\bar{z}) \cdot k) y^{\prime-1} x^{\prime-1} y^{\prime-1} x^{\prime-1}, \\
& b a b^{-1} a^{-1} \mapsto y^{\prime} \bar{z} x^{\prime} y^{\prime-1} x^{\prime-1} \bar{z}^{-1} \\
&=(\bar{z} \cdot j)((-\bar{z}) \cdot(-i) \cdot(-j) \cdot(i) \cdot(j))\left[y^{\prime}, x^{\prime}\right] \\
&= \bar{z}(\bar{z} \cdot j)\left[y^{\prime}, x^{\prime}\right] \\
& a^{-1} b^{-2} a^{-1} \mapsto x^{\prime-1} \bar{z}^{-1} y^{\prime-2} x^{\prime-1} \bar{z}^{-1} \\
&=((-\bar{z}) \cdot(-i))\left((-\bar{z}) \cdot(-i)(-j)^{2}(-i)\right) x^{\prime-1} y^{\prime-2} x^{\prime-1} \\
&=(-\bar{z})(\bar{z} \cdot i) x^{\prime-1} y^{\prime-2} x^{\prime-1}, \\
& a^{2} \mapsto \bar{z} x^{\prime} \bar{z} x^{\prime}=\bar{z}(\bar{z} \cdot i) x^{2}, \\
& a b^{-1} a^{-1} b^{-1} \mapsto \bar{z} x^{\prime} y^{\prime-1} x^{\prime-1} \bar{z}^{-1} y^{\prime-1} \\
&= \bar{z}((-\bar{z}) \cdot(-i) \cdot(-j) \cdot(i)) x^{\prime} y^{\prime-1} x^{\prime-1} y^{\prime-1} \\
&=\bar{z}((-\bar{z}) \cdot j) x^{\prime} y^{\prime-1} x^{\prime-1} y^{\prime-1}, \\
& a b a b^{-1} \mapsto \bar{z} x^{\prime} y^{\prime} \bar{z} x^{\prime} y^{\prime-1} \\
&=\bar{z}(\bar{z} \cdot j \cdot i) x^{\prime} y^{\prime} x^{\prime} y^{\prime-1}=\bar{z}(-\bar{z} \cdot k) x^{\prime} y^{\prime} x^{\prime} y^{\prime-1}, \\
& b^{-2} \mapsto y^{\prime-2} .
\end{aligned}
$$


Now recall that

$$
\begin{aligned}
\bar{z} \cdot i= & \left(-z_{1}-z_{2}-z_{4}\right) q^{\prime}+\left(2 z_{1}+z_{2}-z_{3}+2 z_{4}\right) r^{\prime} \\
& +\left(z_{3}-2 z_{4}\right) s^{\prime}+\left(z_{3}-z_{4}\right) t^{\prime} \\
\bar{z} \cdot k= & \left(z_{1}+z_{2}+z_{3}-z_{4}\right) q^{\prime}+\left(-2 z_{1}-2 z_{2}-2 z_{3}+z_{4}\right) r^{\prime} \\
& +\left(2 z_{1}+2 z_{2}+z_{3}\right) s^{\prime}+\left(2 z_{1}+z_{2}\right) t^{\prime}
\end{aligned}
$$

We also find that

$$
\begin{aligned}
\bar{z} \cdot j & =\left(z_{1} q^{\prime}+z_{2} r^{\prime}+z_{3} s^{\prime}+z_{4} t^{\prime}\right) j \\
& =\left(2 z_{1}+z_{2}(1+i)+z_{3}(i-j)+z_{4}(j+k)\right) j \\
& =z_{1}(2 j)+z_{2}(j+k)+z_{3}(1+k)+z_{4}(-1-i) \\
& =\left(-z_{1}+z_{3}\right) q^{\prime}+\left(2 z_{1}-z_{3}-z_{4}\right) r^{\prime}+\left(-2 z_{1}+z_{3}\right) s^{\prime}+\left(z_{2}+z_{3}\right) t^{\prime}
\end{aligned}
$$

We are now prepared to determine the intersection matrix for the form $\eta_{\phi(s)}$ by representing each entry of the intersection matrix for the form $\eta$ via $\bar{\phi}(s)$. We first note:

$$
\begin{aligned}
& \bar{\phi}(s) \bullet(\bar{z} \cdot i)=\left(\begin{array}{cc}
e^{2 \pi i s\left(z_{3}-z_{4}\right)} & 0 \\
0 & e^{-2 \pi i s\left(z_{3}-z_{4}\right)}
\end{array}\right), \\
& \bar{\phi}(s) \bullet(\bar{z} \cdot j)=\left(\begin{array}{cc}
e^{2 \pi i s\left(z_{2}+z_{3}\right)} & 0 \\
0 & e^{-2 \pi i s\left(z_{2}+z_{3}\right)}
\end{array}\right), \\
& \bar{\phi}(s) \bullet(\bar{z} \cdot k)=\left(\begin{array}{cc}
e^{2 \pi i s\left(2 z_{1}+z_{2}\right)} & 0 \\
0 & e^{-2 \pi i s\left(2 z_{1}+z_{2}\right)}
\end{array}\right) .
\end{aligned}
$$

This means that:

$$
\begin{aligned}
& b^{-1} a^{-1} b^{-1} a^{-1} \mapsto \bar{z}((-\bar{z}) \cdot k) y^{\prime-1} x^{\prime-1} y^{\prime-1} x^{\prime-1} \\
& =\bar{z}((-\bar{z}) \cdot k) y^{\prime-1}\left(y^{\prime} x^{\prime}\right)^{-1} y^{\prime-1} \\
& =\bar{z}((-\bar{z}) \cdot k) y^{\prime-1}\left(\left[x^{\prime}, y^{\prime}\right] x^{\prime} y^{\prime}\right)^{-1} x^{\prime-1} \\
& =\bar{z}((-\bar{z}) \cdot k)\left[x^{\prime}, y^{\prime}\right] y^{\prime-2} x^{\prime-2} \\
& \mapsto\left(\begin{array}{cc}
e^{2 \pi i s z_{4}} & 0 \\
0 & e^{-2 \pi i s z_{4}}
\end{array}\right)\left(\begin{array}{cc}
e^{2 \pi i s\left(-2 z_{1}-z_{2}\right)} & 0 \\
0 & e^{-2 \pi i s\left(-2 z_{1}-z_{2}\right)}
\end{array}\right)\left(\begin{array}{ll}
0 & 1 \\
1 & 0
\end{array}\right) \\
& =\left(\begin{array}{cc}
0 & e^{2 \pi i s\left(-2 z_{1}-z_{2}+z_{4}\right)} \\
e^{-2 \pi i s\left(-2 z_{1}-z_{2}+z_{4}\right)} & 0
\end{array}\right) \text {, } \\
& {[b, a] \mapsto \bar{z}(\bar{z} \cdot j)[y, x]} \\
& \mapsto\left(\begin{array}{cc}
e^{2 \pi i s z_{4}} & 0 \\
0 & e^{-2 \pi i s z_{4}}
\end{array}\right)\left(\begin{array}{cc}
e^{2 \pi i s\left(z_{2}+z_{3}\right)} & 0 \\
0 & e^{-2 \pi i s\left(z_{2}+z_{3}\right)}
\end{array}\right)\left(\begin{array}{ll}
0 & 1 \\
1 & 0
\end{array}\right) \\
& =\left(\begin{array}{cc}
0 & e^{2 \pi i s\left(z_{2}+z_{3}+z_{4}\right)} \\
e^{-2 \pi i s\left(z_{2}+z_{3}+z_{4}\right)} & 0
\end{array}\right) \text {, } \\
& a^{-1} b^{-2} a^{-1} \mapsto(-\bar{z})(\bar{z} \cdot i) x^{\prime-1} y^{\prime-2} x^{\prime-1} \\
& =(-\bar{z})(\bar{z} \cdot i) x^{\prime-1} y^{\prime-1}\left(x^{\prime} y^{\prime}\right)^{-1} \\
& =(-\bar{z})(\bar{z} \cdot i) x^{\prime-1} y^{\prime-1}\left(\left[x^{\prime}, y^{\prime}\right] y^{\prime} x^{\prime}\right)^{-1} \\
& =(-\bar{z})(\bar{z} \cdot i)\left[x^{\prime}, y^{\prime}\right] x^{\prime-1} y^{\prime-1} x^{\prime-1} y^{\prime-1} \\
& =(-\bar{z})(\bar{z} \cdot i)\left[x^{\prime}, y^{\prime}\right] x^{\prime-1}\left(x^{\prime} y^{\prime}\right)^{-1} y^{\prime-1}
\end{aligned}
$$




$$
\begin{aligned}
& =(-\bar{z})(\bar{z} \cdot i)\left[x^{\prime}, y^{\prime}\right] x^{\prime-1}\left(\left[x^{\prime}, y^{\prime}\right] y^{\prime} x^{\prime}\right)^{-1} y^{\prime-1} \\
& =(-\bar{z})(\bar{z} \cdot i) x^{\prime-2} y^{\prime-2} \\
& \mapsto\left(\begin{array}{cc}
e^{2 \pi i s\left(-z_{4}\right)} & 0 \\
0 & e^{-2 \pi i s\left(-z_{4}\right)}
\end{array}\right)\left(\begin{array}{cc}
e^{2 \pi i s\left(z_{3}-z_{4}\right)} & 0 \\
0 & e^{-2 \pi i s\left(z_{3}-z_{4}\right)}
\end{array}\right)\left(\begin{array}{ll}
1 & 0 \\
0 & 1
\end{array}\right) \\
& =\left(\begin{array}{cc}
e^{2 \pi i s\left(z_{3}-2 z_{4}\right)} & 0 \\
0 & e^{-2 \pi i s\left(z_{3}-2 z_{4}\right)}
\end{array}\right) .
\end{aligned}
$$

So the element $1+\left(b^{-1} a^{-1}\right)^{2}-[b, a]-a^{-1} b^{-2} a^{-1}$ goes to the matrix $R(s)$ :

$$
\left[\begin{array}{cc}
1-e^{2 \pi i s\left(z_{3}-2 z_{4}\right)} & e^{2 \pi i s\left(-2 z_{1}-z_{2}+z_{4}\right)}-e^{2 \pi i s\left(z_{2}+z_{3}+z_{4}\right)} \\
e^{-2 \pi i s\left(-2 z_{1}-z_{2}+z_{4}\right)}-e^{-2 \pi i s\left(z_{2}+z_{3}+z_{4}\right)} & 1-e^{-2 \pi i s\left(z_{3}-2 z_{4}\right)}
\end{array}\right] .
$$

Similarly we find that

$$
\begin{aligned}
& a^{2} \mapsto \bar{z}(\bar{z} \cdot i) x^{\prime 2} \\
& \mapsto\left(\begin{array}{cc}
e^{2 \pi i s z_{4}} & 0 \\
0 & e^{-2 \pi i s z_{4}}
\end{array}\right)\left(\begin{array}{cc}
e^{2 \pi i s\left(z_{3}-z_{4}\right)} & 0 \\
0 & e^{-2 \pi i s\left(z_{3}-z_{4}\right)}
\end{array}\right)\left(\begin{array}{ll}
0 & 1 \\
1 & 0
\end{array}\right) \\
& =\left(\begin{array}{cc}
0 & e^{2 \pi i s z_{3}} \\
e^{-2 \pi i s z_{3}} & 0
\end{array}\right) \text {, } \\
& a b^{-1} a^{-1} b^{-1} \mapsto \bar{z}(-\bar{z} \cdot j) x^{\prime} y^{\prime-1} x^{\prime-1} y^{\prime-1} \\
& =\bar{z}(-\bar{z} \cdot j) x^{\prime}\left(x^{\prime} y^{\prime}\right)^{-1} y^{\prime-1} \\
& =\bar{z}(-\bar{z} \cdot j) x^{\prime}\left(\left[x^{\prime}, y^{\prime}\right] y^{\prime} x^{\prime}\right)^{-1} y^{-1} \\
& =\bar{z}(-\bar{z} \cdot j)\left[x^{\prime}, y^{\prime}\right] y^{\prime-2} \\
& \mapsto\left(\begin{array}{cc}
e^{2 \pi i s z_{4}} & 0 \\
0 & e^{-2 \pi i s z_{4}}
\end{array}\right)\left(\begin{array}{cc}
e^{2 \pi i s\left(-z_{2}-z_{3}\right)} & 0 \\
0 & e^{-2 \pi i s\left(-z_{2}-z_{3}\right)}
\end{array}\right)\left(\begin{array}{ll}
1 & 0 \\
0 & 1
\end{array}\right) \\
& =\left(\begin{array}{cc}
e^{2 \pi i s\left(z_{4}-z_{2}-z_{3}\right)} & 0 \\
0 & e^{-2 \pi i s\left(z_{4}-z_{2}-z_{3}\right)}
\end{array}\right) \text {, } \\
& a b a b^{-1} \mapsto \bar{z}(-\bar{z} \cdot k) x^{\prime}\left(y^{\prime} x^{\prime}\right) y^{\prime-1} \\
& =\bar{z}(-\bar{z} \cdot k) x^{\prime}\left(x^{\prime} y^{\prime}\left[x^{\prime}, y^{\prime}\right]\right) y^{\prime-1} \\
& =\bar{z}(-\bar{z} \cdot k)\left[x^{\prime}, y^{\prime}\right] x^{\prime 2} \\
& \mapsto\left(\begin{array}{cc}
e^{2 \pi i s z_{4}} & 0 \\
0 & e^{-2 \pi i s z_{4}}
\end{array}\right)\left(\begin{array}{cc}
e^{2 \pi i s\left(-2 z_{1}-z_{2}\right)} & 0 \\
0 & e^{-2 \pi i s\left(-2 z_{1}-z_{2}\right)}
\end{array}\right)\left(\begin{array}{ll}
1 & 0 \\
0 & 1
\end{array}\right) \\
& =\left(\begin{array}{cc}
e^{2 \pi i s\left(z_{4}-2 z_{1}-z_{2}\right)} & 0 \\
0 & e^{-2 \pi i s\left(z_{4}-2 z_{1}-z_{2}\right)}
\end{array}\right) .
\end{aligned}
$$

So the element $a^{2}+a b^{-1} a^{-1} b^{-1}-a b a b^{-1}-b^{-2}$ goes to the matrix $T(s)$ :

$$
\left[\begin{array}{cc}
e^{2 \pi i s\left(z_{4}-z_{2}-z_{3}\right)}-e^{2 \pi i s\left(z_{4}-2 z_{1}-z_{2}\right)} & -1+e^{2 \pi i s z_{3}} \\
-1+e^{-2 \pi i s z_{3}} & e^{-2 \pi i s\left(z_{4}-z_{2}-z_{3}\right)}-e^{-2 \pi i s\left(z_{4}-2 z_{1}-z_{2}\right)}
\end{array}\right] .
$$

We see that under the representation $\bar{\phi}(s)$, the $4 \times 4$ matrix $P \otimes_{g} \mathbb{Z}[\bar{G}]$ goes to the $8 \times 8$ matrix

$$
U(s)=\left(\begin{array}{cccc}
I_{2 \times 2} & 0_{2 \times 2} & 0_{2 \times 2} & R(s) \\
0_{2 \times 2} & I_{2 \times 2} & T(s) & 0_{2 \times 2} \\
0_{2 \times 2} & {[T(s)]^{H}} & I_{2 \times 2} & 0_{2 \times 2} \\
{[R(s)]^{H}} & 0_{2 \times 2} & 0_{2 \times 2} & I_{2 \times 2}
\end{array}\right)
$$


where $H$ denotes taking the complex conjugate transpose. Thus, under $\bar{\phi}(s)$ the $8 \times 8$ matrix $Q \otimes_{g} \mathbb{Z}[\bar{G}]$ goes to the $16 \times 16$ matrix

$$
V(s)=\left(\begin{array}{cc}
2 U(s) & 3 I_{8 \times 8} \\
3 I_{8 \times 8} & 4 U(s)
\end{array}\right) .
$$

When $s=0$, we simply have the matrix

$$
V(0)=\left(\begin{array}{ll}
2 I_{8 \times 8} & 3 I_{8 \times 8} \\
3 I_{8 \times 8} & 4 I_{8 \times 8}
\end{array}\right)
$$

whose signature is zero. In summary

$$
\sigma(\eta) \bullet \bar{\phi}(0)=\operatorname{signature}\left(\eta_{\bar{\phi}(0)}\right)=\operatorname{signature}(V(0))=0 .
$$

When $s=1$, we have the matrix $V(1)$. We compute the signature of $V(1)$ numerically and determine that

$$
\sigma(\eta) \bullet \bar{\phi}(1)=\operatorname{signature}\left(\eta_{\bar{\phi}(1)}\right)=\operatorname{signature}(V(1))=4 \neq 0 .
$$

So we have shown that $\sigma(\eta) \circ \bar{\phi}: \mathbb{R} \rightarrow \mathbb{R}$ does not have period 1, which means $\bar{\sigma}\left(\widetilde{M},\left.p\right|_{\bar{J}} \circ \widetilde{\alpha}\right) \circ \bar{\phi}: \mathbb{R} \rightarrow \mathbb{R}$ does not have period 1 , which means that the $\bar{G}-$ structure on $\widetilde{M}$ does not come from a $G$-structure. Hence the examples, $f$, of Lemma 6.1 are noncharacteristic. Since the signature is a cobordism invariant on $\operatorname{im}(\bar{\phi})$, the maps $f$ are not cobordant to characteristic embeddings

In Chapter 2 of [CS1], it is shown that if $\mathscr{G}: \mathbb{Z}[\pi] \rightarrow \Lambda$ is onto, then $\Gamma_{2 k+1}(\mathscr{G})$ is a subgroup of $L_{2 k+1}(\Lambda)$. Consider the commutative diagram:

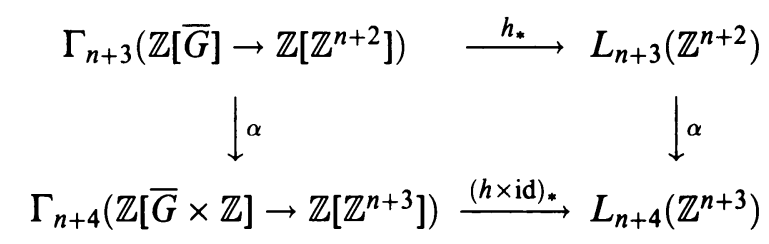

where $\alpha$ is the map that corresponds geometrically to taking cartesian products with a circle. Since $n+4$ is odd, the lower horizontal map is the inclusion of a subgroup. Let $\delta_{n} \in \Gamma_{n+3}(\bar{F})$ be the element represented by a form with the intersection matrix

$$
\left(\begin{array}{ll}
2 & 3 \\
3 & 4
\end{array}\right) .
$$

Then the realization theorem for $\Gamma$ groups associates to $\delta_{n}$ a $G$-manifold $N$ and, by the above diagram,

$$
\alpha\left(\delta_{n}\right)=\alpha\left(\gamma_{n}\right) .
$$

Applying the realization theorem for $\Gamma$-groups to this element we find that $M \times S^{1}$ is contained in the same $\mathbb{Z}^{n+3}$-homology $\bar{G}$-bordism class as the $G \times \mathbb{Z}$ manifold $N \times S^{1}$. This means that if $f$ is one of the examples in Lemma 4.1, $f \times \mathrm{id}_{S^{1}}: T^{n+1} \rightarrow T^{n+3}$ is cobordant to a characteristic embedding.

\section{CONCLUDING REMARKS}

The adaptation of the methods of [LD] to the study of embeddings $f: T^{n} \rightarrow$ $T^{n+2}$ holds out promise for complete cobordism classification in all dimensions. It is possible, for example, that the set of cobcrdism classes in even dimensions contains only one element. 


\section{REFERENCES}

[APS] M. F. Atiyah, V. K. Patodi, and I. M. Singer, Spectral asymmetry and Riemannian geometry. II, Math. Proc. Cambridge Philos. Soc. 78 (1975), 405-432.

[B1] A. K. Bousfield, Homological localization towers for groups and П-modules, Mem. Amer. Math. Soc., vol. 10, no. 186 (1977).

[B2] — The localization of spaces with respect to homology, Topology 14 (1975), 133-150.

[CS1] S. E. Cappell and J. L. Shaneson, The codimension two placement problem and homology equivalent manifolds, Ann. of Math. (2) 99 (1974), 277-348.

[CS2] _ Link cobordism, Comment. Math. Helv. 55 (1980), 20-49.

[CS3] __ An introduction to embeddings, immersions and singularities in codimension two, Proc. Sympos. Pure Math., vol. 32, Amer. Math. Soc., Providence, R.I., 1978, pp. 129-149.

[CO1] T. D. Cochran and K. E. Orr, Not all links are concordant to boundary links, Bull. Amer. Math. Soc. 23 (1990), 99-106.

[CO2] _ Not all links are concordant to boundary links, preprint.

[HS] Wu-Chung Hsiang and J. L. Shaneson, Fake tori, Topology of Manifolds (J. C. Cantrell and C. H. Edwards, eds.), Markham, Chicago, Ill., 1970, pp. 18-51.

[LD] J. Le Dimet, Cobordisme d'enlacement de disques, Mem. Math. France 116 (1988).

[L1] J. P. Levine, Finitely-presented groups with long lower central series, preprint.

[L2] _ Link concordance and algebraic closure. II, Invent. Math. 96 (1989), 571-592.

[L3] _ Signature invariants of homology bordism with applications to links, Proc. Osaka Knot Theory Conf.

[L4] Link concordance and algebraic closure of groups, Comment. Math. Helv 64 (1989), 236-255.

[L5] _ Algebraic closure of groups, Combinatorial Group Theory, Contemp. Math., vol. 109, Amer. Math. Soc., Providence, R.I., 1990, pp. 99-106.

[L6] L Link invariants via the eta invariant, preprint.

[V] Pierre Vogel, Localization of spaces with respect to a class of maps, preprint.

[W] C. T. C. Wall, Surgery on compact manifolds, Academic Press, London and New York, 1970.

Department of Mathematics, Swarthmore College, Swarthmore, Pennsylvania 19081

Current address: Department of Mathematics, William Patterson College, Wayne, New Jersey 07470 